Math. Model. Nat. Phenom.

Vol. 4, No. 6, 2009, pp. 54-90

DOI: $10.1051 / \mathrm{mmnp} / 20094602$

\title{
Evolutionary Games in Space
}

\author{
N. Kronik ${ }^{1}$, Y. Cohen ${ }^{2 *}$ \\ ${ }^{1}$ Department of Applied Mathematics, Holon Institute of Technology, Holon 58102, Israel \\ ${ }^{2}$ Department of Fisheries, Wildlife, and Conservation Biology \\ University of Minnesota, St. Paul, MN 55118
}

\begin{abstract}
The $G$-function formalism has been widely used in the context of evolutionary games for identifying evolutionarily stable strategies (ESS). This formalism was developed for and applied to point processes. Here, we examine the $G$-function formalism in the settings of spatial evolutionary games and strategy dynamics, based on reaction-diffusion models. We start by extending the point process maximum principle to reaction-diffusion models with homogeneous, locally stable surfaces. We then develop the strategy dynamics for such surfaces. When the surfaces are locally stable, but not homogenous, the standard definitions of ESS and the maximum principle fall apart. Yet, we show by examples that strategy dynamics leads to convergent stable inhomogeneous strategies that are possibly ESS, in the sense that for many scenarios which we simulated, invaders could not coexist with the exisiting strategies.
\end{abstract}

Key words: mathematical modeling, game theory, reaction-diffusion equation, $G$-function, evolutionary ecology

AMS subject classification: 35B30, 35B20, 91A22

\section{Introduction}

The study of dynamic systems with inheritance has a long and venerable history (see, e.g, Gorban [24] for an overview). The analysis of evolutionary processes as a game among players with different inherited strategies was first introduced by Maynard-Smith and Price [37]. A strategy is a value of some organism's trait that influences the organism's fitness. This inherited strategy can be

${ }^{*}$ Corresponding author. E-mail: natalieka@ hit.ac.il 
a behavioral phenotype, e.g., whether to fight or flee upon a challenge, or an adaptive parameter, e.g., age at first reproduction or the relative number of sons and daughters produced by a parent [36].

A key concept in applying game theory to evolutionary processes is the evolutionarily stable strategy (ESS). An ESS is defined as a (pure or mixed) strategy, such that if all members of a population adopt it, then no mutant strategy can survive indefinitely under the influence of natural selection [36]. A mutant strategy is a rare alternative strategy different from that of the ESS. This definition guarantees the long-term survival of the evolutionarily stable strategies, even if those do not result in maximal fitness $[59,56]$. We note that here we follow the modern ESS terminology, but a surviving strategy that is uninvadible by rare mutant strategies was discussed as early as the '30s of the previous century [28, 20]. The concept of an evolutionary game ending in ESS values has found much use in analysis of adaptive parameters in co-evolving populations within a species and between species $[29,49,2,5]$ and in species richness analysis $[54,8,55,57,58,5,12,11]$.

Often, use of the ESS concept tacitly assumes that a system which allows for mutations will eventually arrive at the ESS values under natural selection, regardless of its starting point. This does not address, however, the issue of how it would get to ESS. By analyzing strategy dynamics (and not just the static, stable strategies) Eshel and Motro [19] and Takada and Kigami [48] pointed out that perturbation around the ESS does not necessarily lead to a return to ESS value. Eshel [17] distinguished between "evolutionary unbeatable strategy" and "continuous stability", Taylor [49] introduced " $\delta$-stability" and " $m$-stability", and Apaloo [5] introduced the "neighbourhood invader strategy" to describe resistance to invasion and convergence stability. Following Eshel [18], we shall refer to strategies for which small deviations lead to an evolutionary disadvantage, whereas a return to the exact strategy value leads to an evolutionary advantage as convergent-stable (CS). A considerable effort was devoted to various mathematical formulations of conditions under which strategies that are ESS in the Maynard-Smith sense are also CS [19, 49, 58, 2, 18, 23], or (as elaborated later) to redefining the ESS concept such that it inherently includes the CS property [12].

Vincent and co-workers (see Ref. [59], and references therein) developed a mathematical formalism for predicting the number and values of the pure strategies that comprise the ESS. This formalism is based on a fitness generating function ( $G$-function) that can be used to analyze population and strategy dynamics. Furthermore, by using a so-called ESS maximum principle (discussed in detail below) and strategy dynamics we ensure that the strategies found are convergent stable. This approach has been applied to the Lotka-Volterra competition model, the Monod competition model, and predator-prey systems $[9,12,56]$.

Traditionally, the theory of evolutionary games in general, and the generating function approach in particular, do not include the possible effects of spatial population variations on the outcome of the game. Indeed, the $G$-function approach was developed for and applied to point processes. However, it is well known that spatial effects can and do affect numerous biological and ecological properties, such as the progress of epidemics, pattern formation in animals, and habitat fragmentation $[39,50]$. Vickers [52] has noted that because the concept of ESS inherently contains mutant strategies, which may involve migrating groups, a mathematical treatment allowing for spatial variation is required. He suggested the addition of a diffusion term to the "reaction 
matrix" describing the evolutionary game as an appropriate recourse. In early studies based on this reaction-diffusion approach $[52,30,53]$, Vickers and co-workers have shown that the ESS is locally stable to small spatial perturbations and will not give rise to spatial patterns. However, later work [13] has shown that for some matrix forms a spatial pattern may emerge.

Reaction-diffusion equations are relatively straightforward to construct and permit complex patterns formation $[47,6,25]$. They have been used extensively in other contexts of spatial pattern formation, such as chemical reactions in open systems [42] and morphogenesis [39, and references therein]. This suggests a need for an extension of the ESS concept in general, and the $G$-function formalism in particular, including strategy dynamics, to the case of spatially varying populations. To the best of our knowledge, this has not been attempted.

Here, we present a generalization of the $G$-function approach to spatial processes. Our beginning is modest: we deal with constant diffusion coefficients (the diffusion rates are not subject to evolution) with scalar strategies and within a closed space; i.e., no migration across boundaries.

In Section 2, we set the stage by defining the problem in mathematical terms and by providing the necessary conditions for a homogeneous, locally stable ESS to exist. This is achieved by proving a generalization of the maximum principle of Vincent et al. [59, 56]. In Section 3, we develop a spatial generalization to strategy dynamics and pursue the CS properties of spatially homogeneous steady state solutions, based on the spatial $G$-function generalization developed in Section 2. We end the section with numerical examples. In Section 4 we discuss the case of an inhomogeneous steady-state solution. We show how this case is intimately related to the concept of diffusion-driven (Turing) instability. We explore possible ESS properties of CS strategies in this general case, with the aid of numerical examples. Finally, concluding remarks and suggestions for further research are given in Section 5.

\section{ESS for a spatially homogeneous equilibrium}

In this section, we extend the point process $G$-function formalism by extending the point process work of Vincent and co-workers $[59,56]$ to the case of a spatially homogeneous equilibrium. We define an ecological equilibrium and an evolutionary equilibrium within a reaction-diffusion model, derive a maximum principle, and derive sufficient conditions for a spatially homogeneous ESS to exist. The concepts are then illustrated with numerical solutions.

\subsection{Problem statement}

Consider a community of $N$ species in an $n$ dimensional (n being 1,2 , or 3 ) bounded domain $\Omega$ with coordinates $\mathbf{x}:=\left[x_{1}, \ldots, x_{n}\right]$. Denote by $z_{i}(\mathbf{x}, t), t \geq 0$ the population density of species $i$, $i=1, \ldots, N$ at location $\mathbf{x}$ at time $t$, and by $u_{i}$ the strategy value of species $i$. Assume that $u_{i} \in \Re$. To begin with, assume that $u_{i}$ is a constant. Let $\mathbf{u}:=\left[u_{1}, \ldots, u_{N}\right]$ and $\mathbf{z}:=\left[z_{1}, \ldots, z_{N}\right]$.

Reaction functions $f_{i}(\mathbf{u}, \mathbf{z}), i=1, \ldots, N$, describe the birth and death rates of the populations. Next, assume that migration (diffusion) is proportional to a population gradient over $\mathbf{x}$ with a 
proportionality coefficient $d_{i}>0$ and that species migrate independently of each other. We retain this assumption for the remainder of this paper. Let

$$
\Delta=\frac{\partial^{2}}{\partial x_{1}^{2}}+\cdots+\frac{\partial^{2}}{\partial x_{n}^{2}},
$$

where $n=1,2$, or 3 , and

$$
\mathbf{D}=\operatorname{diag}\left[d_{1}, \ldots, d_{N}\right]
$$

That is, $\mathbf{D}$ is a diagonal matrix with diffusion coefficients $d_{i}$ on the diagonal. Let $\partial_{t}:=\frac{\partial}{\partial t}$. The dynamics of species $i$ is modeled with

$$
\partial_{t} z_{i}(\mathbf{x}, t)=f_{i}(\mathbf{u}, \mathbf{z}(\mathbf{x}, t))+d_{i} \Delta z_{i}(\mathbf{x}, t), \quad i=1,2, \ldots, N .
$$

The system is written as

$$
\partial_{t} \mathbf{z}(\mathbf{x}, t)=\mathbf{f}(\mathbf{u}, \mathbf{z}(\mathbf{x}, t))+\mathbf{D} \Delta \mathbf{z}(\mathbf{x}, t) .
$$

Systems of the form (2.2) are known as reaction-diffusion equations [51, 47, 42, 10, 25]. Traditionally, the boundary of the spatial domain is denoted by $\partial \Omega$ (here $\partial \Omega$ should be understood as a single symbol; $\partial$ is not an operator). $\bar{\Omega}$ designates the spatial domain, including its boundary. If it exists, the solution $\mathbf{z}$ is defined in the spatial domain $\bar{\Omega}$ and for time 0 to $\infty$. To state the problem correctly, we must specify conditions on the boundaries (these must be satisfied at all times) and initial conditions. The initial conditions give the distribution of $\mathbf{z}$ over the spatial domain at $t=0$. A solution must be consistent with the initial and boundary conditions.

We deal with processes where there is no migration across the boundary. This means that there is no flow (flux) across $\partial \Omega$. This fact is described as follows: The perpendicular to a tangent through a point on the boundary, when pointing outside the domain, describes the direction of flow across the spatial domain from inside to outside. This perpendicular of unit length is called the outward normal. At each point on the boundary, take the derivative of $z_{i}$ in the direction of the normal. This derivative describes the flow of $\mathbf{z}$ as a function of $\mathbf{x}$ at a point on the boundary. To ensure that there is no flux across the whole boundary, the derivative of $\mathbf{z}$ on the boundary in the direction of the outside pointing normal must be zero. This condition of no migration across the boundary must be true for all $t \geq 0$. Biologically, this is equivalent to the case of fenced animals that are unable to cross the fence, or to animals on an island that are unable to get off the island. Therefore we write

$$
\frac{\partial \mathbf{z}}{\partial n}(\mathbf{x}, t)=\mathbf{0} \quad \text { on } \quad \partial \Omega \times[0, \infty),
$$

where $n$ is the outwards pointing normal. This is called the Neumann boundary condition with zero flux [32]. The initial conditions are given by

$$
\mathbf{z}(\mathbf{x}, 0)=\mathbf{z}_{0}(\mathbf{x}) \quad \text { on } \Omega \times\{0\},
$$

where $\mathbf{z}_{0}(\mathbf{x})$ is given. We are interested in a special form of $\mathbf{f}$ :

$$
f_{i}(\mathbf{u}, \mathbf{z}(\mathbf{x}, t))=z_{i}(\mathbf{x}, t) H_{i}(\mathbf{u}, \mathbf{z}(\mathbf{x}, t))
$$


This allows us to explore the role of the instantaneous fitness functions $H_{i}$. Equation (2.2) with the boundary condition (2.3) and initial condition (2.4) define our problem. We denote it by $P_{E}$. Note that our model assumes that $\mathbf{u}$ is constant in space (spatially homogeneous), an assumption we relax in Section 3.

Under certain conditions, we expect that the solution of $P_{E}$ approaches a steady state. Thus, we have

Definition 1 (equilibrium solution). A solution $\overline{\mathbf{z}}(\mathbf{x})$ of $P_{E}$ which satisfies

$$
\bar{z}_{i}(\mathbf{x}, t) H_{i}(\mathbf{u}, \overline{\mathbf{z}}(\mathbf{x}, t))+d_{i} \Delta \bar{z}_{i}(\mathbf{x}, t)=0, i=1, \ldots, N
$$

is called the equilibrium solution.

If the equilibrium solution is homogeneous in space, $\Delta \bar{z}_{i}(\mathbf{x}, \mathbf{t})=0$ and therefore $\bar{z}_{i} H_{i}=0$. If $\bar{z}_{i}=0$ for all $i$, the solution is trivial. If $\bar{z}_{i}>0$ For at least one index $i$, then $H_{i}=0$ and, for reasons clarified below, this is called a maximum fitness solution.

\subsection{Ecologically stable equilibrium}

At equilibrium, the left hand side of Eq. (2.2) must be zero. We will refer to an equilibrium as an ecologically stable one if it implies the survival of at least one species. Formally:

Definition 2 (spatial ecological equilibrium). Given the strategy vector $\mathbf{u}$, and an equilibrium solution $\bar{z}_{i}(\mathbf{x})$ of $P_{E}, \bar{z}_{i}(\mathbf{x})$ is an ecological equilibrium for $P_{E}$ if, for any $\mathbf{x} \in \bar{\Omega}$, there exists $1 \leq \sigma \leq N$ such that

$$
\begin{aligned}
\bar{z}_{i}(\mathbf{x}) \geq 0 \quad \text { and } \quad \bar{z}_{i}(\mathbf{x}) H_{i}(\mathbf{u}, \overline{\mathbf{z}}(\mathbf{x}))+d_{i} \Delta \bar{z}_{i}(\mathbf{x})=0, & i=1, \ldots, \sigma \\
\bar{z}_{i}(\mathbf{x})=0, & i=\sigma+1, \ldots, N
\end{aligned}
$$

and for $i=1, \ldots, \sigma, \bar{z}_{i}(\mathbf{x})>0$ for some domain $\hat{\Omega} \subseteq \bar{\Omega}$.

This definition is a modification of the definition of Vincent et al. [59] for a point process ecological equilibrium. As for the point-process case, a spatial ecological equilibrium $\overline{\mathbf{z}}(\mathbf{x})$ may or may not be locally stable. The definition of a locally stable spatial ecological equilibrium is given in Appendix 1 of Ref. [31]. It implies that the linearized stability of $P_{E}$ around the zero solution of $P_{E}$ is also the local stability of the non-linear problem $P_{E}$ [10].

Having defined a locally stable ecological equilibrium for the spatial case, the question is how to find practical criteria for the local stability of an ecological equilibrium. To answer that, we first extend the point process analysis to that of the homogeneous equilibrium solution $(\overline{\mathbf{z}})$ of $P_{E}$. An analysis of the general case is provided in Section 3 below. We suppose that an equilibrium solution to $P_{E}$ exists and define $\mathbf{w}(\mathbf{x}, t) \triangleq \mathbf{z}(\mathbf{x}, t)-\overline{\mathbf{z}}$. It is well known [51] that, in general, one cannot expect the stability properties of the equilibrium solution of $P_{E}$ to be the same as the stability properties of the corresponding system without diffusion:

$$
\frac{\partial \mathbf{z}(t)}{\partial t}=\mathbf{z}(t) \mathbf{H}(\mathbf{u}, \mathbf{z}(t))
$$


One exception is when all of the diffusion coefficients are equal [27]. As this exception is highly restrictive, we shall not pursue it.

The (linearized) perturbation equation for a spatially homogeneous equilibrium solution of $P_{E}$ is now

$$
\begin{aligned}
\frac{\partial \mathbf{w}(\mathbf{x}, t)}{\partial t} & =\mathbf{A} \mathbf{w}(\mathbf{x}, t)+\mathbf{D} \Delta \mathbf{w}(\mathbf{x}, t), \quad x \in \Omega, \quad t>0, \\
\frac{\partial \mathbf{w}(\mathbf{x}, t)}{\partial n} & =0 \quad \text { on } \quad \partial \Omega \times[0, \infty), \\
\mathbf{w}(\mathbf{x}, 0) & =\mathbf{w}_{0},
\end{aligned}
$$

where $\mathbf{A}$ is an $N \times N$ matrix with elements

$$
\left.a_{i, j} \triangleq \frac{\partial\left[z_{i} H_{i}(\mathbf{u}, \mathbf{z})\right]}{\partial z_{j}}\right|_{\mathbf{z}=\overline{\mathbf{z}}} .
$$

Note that derivatives are first taken and then the values of $z_{i}$ are replaced by $\bar{z}_{i}$.

It can be shown $[34,10,25]$ that with zero flux boundary conditions, there is an infinite number of stability matrices of the linearized problem (2.8), namely

$$
\mathbf{A}_{k}=\left[\mathbf{A}-\lambda_{k} \mathbf{D}\right], \quad k=0,1, \ldots,
$$

where $\lambda_{k}$ are eigenvalues that obey:

$$
\triangle \phi_{k}=\lambda_{k} \phi_{k}, \quad k=0,1, \ldots
$$

and $\phi_{k}$ are the corresponding eigenfunctions. Here

$$
0=\lambda_{0} \leq \lambda_{1} \leq \lambda_{2} \leq \ldots
$$

Because of the dependence on boundary conditions, the values of $\lambda_{k}$, and therefore the stability of the system, depend on the size and shape of the domain. The solution of (2.8) is locally stable asymptotically if and only if the eigenvalues for all $\mathbf{A}_{k}$ have negative real parts. As we shall see next, these matrices have a particularly simple form in our case.

Based on Definition 2, and following Vincent et al. (1996), we partition $\mathbf{A}_{k}$ as follows:

$$
\mathbf{A}_{k}=\left[\begin{array}{ll}
\left(\mathbf{A}_{1}\right)_{k} & \left(\mathbf{A}_{2}\right)_{k} \\
\mathbf{0} & \left(\mathbf{A}_{3}\right)_{k}
\end{array}\right],
$$

where

$$
\left(\mathbf{A}_{1}\right)_{k}=\left[\begin{array}{lll}
\left.\bar{z}_{1} \frac{\partial H_{1}}{\partial \bar{z}_{1}}\right|_{\bar{z}}-\lambda_{k} d_{1} & \cdots & \left.\bar{z}_{1} \frac{\partial H_{1}}{\partial \bar{z}_{\sigma}}\right|_{\bar{z}} \\
\vdots & \ddots & \vdots \\
\left.\bar{z}_{\sigma} \frac{\partial H_{\sigma}}{\partial \bar{z}_{1}}\right|_{\bar{z}} & \cdots & \left.\bar{z}_{\sigma} \frac{\partial H_{\sigma}}{\partial \bar{z}_{\sigma}}\right|_{\bar{z}}-\lambda_{k} d_{\sigma}
\end{array}\right]
$$




$$
\begin{array}{r}
\left(\mathbf{A}_{2}\right)_{k}=\left[\begin{array}{lll}
\left.\bar{z}_{1} \frac{\partial H_{1}}{\partial \bar{z}_{\sigma+1}}\right|_{\bar{z}} & \cdots & \left.\bar{z}_{1} \frac{\partial H_{1}}{\partial \bar{z}_{N}}\right|_{\bar{z}} \\
\vdots & \ddots & \vdots \\
\left.\bar{z}_{\sigma} \frac{\partial H_{\sigma}}{\partial \bar{z}_{\sigma+1}}\right|_{\bar{z}} & \cdots & \left.\bar{z}_{\sigma} \frac{\partial H_{\sigma}}{\partial \bar{z}_{N}}\right|_{\bar{z}}
\end{array}\right], \\
\left(\mathbf{A}_{3}\right)_{k}=\left[\begin{array}{lll}
H_{\sigma+1}-\lambda_{k} d_{\sigma+1} & \cdots & 0 \\
\vdots & \ddots & \vdots \\
0 & \cdots & H_{N}-\lambda_{k} d_{N}
\end{array}\right] .
\end{array}
$$

$A_{k}$ is an upper block-triangular matrix. The determinant of a block diagonal matrix is equal to the product of the determinants of its diagonal blocks [35]. Therefore, $\operatorname{det}(\lambda I-A)_{k}=\operatorname{det}(\lambda I-$ $\left.A_{1}\right)_{k} \operatorname{det}\left(\lambda I-A_{3}\right)_{k}$. This indicates that the stability of $\mathbf{A}_{k}$ is determined by the eigenvalues of $\left(\mathbf{A}_{1}\right)_{k}$ and $\left(\mathbf{A}_{3}\right)_{k}$ only. Based on Definition 2, $\left(\mathbf{A}_{1}\right)_{k}$ are matrices describing the "surviving" species, whereas $\left(\mathbf{A}_{3}\right)_{k}$ are matrices describing the invading (mutant) species. The latter are the species that become extinct because their strategy values cannot coexist with those of the surviving species. With this partition of $\mathbf{A}_{k}$ we have

Proposition 1. Given $P_{E}$, a homogeneous spatial ecological equilibrium is locally stable if all of the real parts of the eigenvalues of $\left(\mathbf{A}_{1}\right)_{k}, k=0,1, \ldots$ are negative and

$$
H_{j}(\mathbf{u}, \overline{\mathbf{z}})<0, \quad j=\sigma+1, \ldots, N .
$$

Proof. The first $\sigma$ eigenvalues of $\mathbf{A}_{k}$ are computed from $\left(\mathbf{A}_{1}\right)_{k}$ only, whereas the last $N-\sigma$ are computed from $\left(\mathbf{A}_{3}\right)_{k}$ only. The solution of the linearized problem is a sum of terms, each of which is exponential in $\left(\lambda_{k} t\right)$. Therefore, for a small perturbation on the linearized system to decay, all of the real parts of these eigenvalues, which are its diagonal elements [4], must be negative. Because $\left(\mathbf{A}_{3}\right)_{k}$ is diagonal, its eigenvalues are $H_{j}(\mathbf{u}, \overline{\mathbf{z}})-\lambda_{k} d_{j}$. However, because $\lambda_{k} d_{j} \geq 0$ it is enough to require only $H_{j}(\mathbf{u}, \overline{\mathbf{z}})<0$. If any of the eigenvalues of $\left(\mathbf{A}_{1}\right)_{k}$ or $\left(\mathbf{A}_{3}\right)_{k}$ is positive, the perturbation solution (the solution of the linearized system) will grow exponentially. If any of the real parts of the eigenvalues of $\mathbf{A}_{k}$ are zero, then further analysis is necessary.

An immediate biological interpretation of the preceding Proposition is that for a single species, the faster a species disperses, the faster it will return to its equilibrium value.

We now discuss the biological implications of the preceding proposition. An important consequence of Proposition 1 is that when a perturbation is spatially homogeneous, we have only $\lambda_{0}=0$, and the proposition reduces to the standard non-spatial case. However, when the perturbation is not spatially homogeneous, $\lambda_{k}$ other than $\lambda_{0}$ come into play and the stability of $\left(\mathbf{A}_{1}\right)_{k}$ cannot be reduced to the case without diffusion and does not necessarily follow from the stability of $\left(\mathbf{A}_{1}\right)_{0}$. If at least one of the $\left(\mathbf{A}_{1}\right)_{k}$ matrices has at least one eigenvalue with a positive real part, then the homogeneous solution is not locally stable, and the system will evolve towards a different, possibly inhomogeneous equilibrium (this case is pursued in detail in Section 4). In fact, much work outside the context of ESS has been dedicated to the problem of the effect of spatial perturbations and diffusion processes on the stability of reacting species [51, 42, 44, 45]. This work stemmed from Turing's initial observation that systems locally stable to spatially homogeneous 
perturbations may or may not be locally stable when the perturbations are not homogeneous. As noted above, the eigenvalues, $\lambda_{k}$, generally depend on the size and shape of the domain. Biologically, this means that the same perturbation may destabilize the system in different ways because of geometrical effects alone. For example, one can imagine that having zero flux on the boundary in a one dimensional domain will result in an entirely different profile for a domain of $(0-\pi)$ and of $(0-2 \pi / 3)$. This issue is elaborated in Section 4. Indeed, Murray [39] dedicates an entire chapter of his book to show how different domain sizes create different biological pattrens. For example, he suggests that the size of the embryo at which diffusion-induced instability occurs determines its final coat pattern: stripes, spots, etc. From an evolutionary ecology standpoint, this further supports the well-known ecological fragmentation problem, namely, that changing the habitat size and shape may lead to a different set of winning strategies and may upset the ecological balance even if no other changes take place [15, 33, 40, 41].

In the following sections, we assume that the real parts of the eigenvalues of all $\left(\mathbf{A}_{1}\right)_{k}$ matrices, diagonal or not, are negative (this will be our sufficient condition), so that the spatially homogeneous ecological equilibrium is locally stable. Our next question is then: Given a spatially homogeneous ecological stability among those species whose densities are positive, what are the conditions that do not allow invading species that may possess a spatially inhomogeneous density to coexist indefinitely?

\subsection{Evolutionarily stable equilibrium}

To move from ecological to evolutionary equilibrium, following Vincent et al. [59], we define:

$$
\begin{gathered}
\mathbf{u}_{c}=\left[u_{1}, \ldots, u_{\sigma}\right], \\
\mathbf{u}_{m}=\left[u_{\sigma+1}, \ldots, u_{N}\right],
\end{gathered}
$$

where the subscripts $c$ and the $m$ denote the vector of existing strategies and the vector of mutant strategies.

Definition 3 (evolutionarily stable strategy). The vector $\mathbf{u}_{c}$ is an ESS for the spatially homogeneous ecological equilibrium $\overline{\mathbf{z}}$ if, for any $N \geq \sigma$ and any $\mathbf{u}_{m}, \overline{\mathbf{z}}$ is a locally stable ecological equilibrium.

The definition implies that once the "right" coalition is found (i.e., both $\sigma$ and the values of $u_{i}$ in $\mathbf{u}_{c}$ have been determined), no other strategy value that invades the community may coexist with $\mathbf{u}_{c}$ indefinitely. Note that Definition 3 is a generalization of Maynard-Smith's original definition. It implies convergent stability as well as resistance to invasion, because the equilibrium is required to be locally stable irrespectively of $N$ [12].

Next, following Vincent et al. [59] we define the $G$-function.

Definition 4 (G-function). A function $G(v, \mathbf{u}, \mathbf{z}(\mathbf{x}, t))$ is a $G$-function for $P_{E}$ if, for every $i=$ $1, \ldots, N$,

$$
G\left(u_{i}, \mathbf{u}, \mathbf{z}(\mathbf{x}, t)\right)=H_{i}(\mathbf{u}, \mathbf{z}(\mathbf{x}, t))
$$


We write the $G$-function in terms of a virtual strategy $v$ as $G(v, \mathbf{u}, \mathbf{z}(\mathbf{x}, t))$. Replacing $v$ by the strategy $u_{i}$ used by individuals of strategy of type $i$ results in the instantaneous fitness function for these individuals. Thus, $v$ represents the strategy of an arbitrary individual while the pair $\mathbf{u}, \mathbf{z}$ define the "current biotic environment". Note that $G$ is a function of the virtual strategy, the current strategies of all species, and the population densities of all species at all locations $\mathbf{x}$. In this sense, $G$ is the spatial fitness generating function.

Theorem 1 (ESS maximum principle). Let $G(v, \mathbf{u}, \mathbf{z})$ be a $G$-function for $P_{E}$. If $\mathbf{u}_{c}=\left[\bar{u}_{1}, \ldots, \bar{u}_{\sigma}\right]$ is an ESS for the locally stable spatially homogeneous ecological equilibrium $\overline{\mathbf{z}}$, then

$$
\begin{aligned}
0 & =\left.G(v, \overline{\mathbf{u}}, \overline{\mathbf{z}})\right|_{v=\bar{u}_{i}}>\left.G(v, \overline{\mathbf{u}}, \overline{\mathbf{z}})\right|_{v=\bar{u}_{j}}, \quad \text { for } \\
i & =1, \ldots, \sigma, \quad j=\sigma+1, \ldots, N
\end{aligned}
$$

must take on its maximum value at $v=\bar{u}_{1}, \ldots, \bar{u}_{\sigma}$.

Proof. From Definition 2,

$$
\bar{z}_{i}>0, \quad \bar{z}_{i} H_{i}(\overline{\mathbf{u}}, \overline{\mathbf{z}})+d_{i} \Delta \bar{z}_{i}(\mathbf{x})=0, \quad i=1, \ldots, \sigma .
$$

From the requirement of a spatially homogeneous equilibrium we have

$$
d_{i} \Delta \bar{z}_{i}(\mathbf{x})=0 .
$$

Therefore, by definition,

$$
G\left(\bar{u}_{i}, \overline{\mathbf{u}}, \overline{\mathbf{z}}\right)=0 .
$$

Because an ESS is a locally stable spatially homogeneous ecological equilibrium, we have from Proposition 1 that

$$
H_{j}(\overline{\mathbf{u}}, \overline{\mathbf{z}})<0, \quad j=\sigma+1, \ldots, N, \quad k=0,1, \ldots
$$

Therefore, by definition,

$$
G\left(\bar{u}_{j}, \overline{\mathbf{u}}, \overline{\mathbf{z}}\right)<0
$$

Consequently, (2.13) must be true.

Note that theorem 1 gives sufficient conditions for a homogeneously stable spatial ESS. Thus, finding the values of $\mathbf{v}$ that maximize $G$ does not guarantee that an ESS has been found. It merely indicates that a candidate solution has been found. Other methods (convergence stability) need to be used to check that a candidate solution is indeed an ESS solution [12].

For species with different ecological roles, each possesing its respective $G$-function with a respective maximum principle, it is easy to show that Theorem 1 also holds. For an extension to such systems with multiple $G$-functions see Appendix 2 of Ref. [31].

It can also be shown that If $\mathbf{u}_{c}$ is an ESS for the locally stable spatially homogeneous ecological equilibrium $\overline{\mathbf{z}}$, then larger diffusion coefficients, or a larger $k$ index of the spatial profile, for the invading species with mutant strategies may only accelerate their extinction. 


\subsection{The Lotka-Volterra competition model: numerical example}

In this section, we illustrate the results developed in the previous sections by means of the LotkaVolterra competition model.

In this model, all species share the same mathematical form of the fitness function, only with different parameters. Therefore, one $G$-function suffices to describe the entire system [59]. We chose the following form for the fitness generating function [12]:

$$
G(v, \mathbf{u}, \mathbf{z})=r-\frac{r}{k(v)} \sum_{j=1}^{\sigma} \alpha\left(v, u_{j}\right) z_{j},
$$

The fitness function is:

$$
H_{i}(\mathbf{u}, \mathbf{z})=r-\frac{r}{k\left(u_{i}\right)} \sum_{j=1}^{\sigma} \alpha\left(u_{i}, u_{j}\right) z_{j}
$$

where

$$
k\left(u_{i}\right)=k_{m} \exp \left(\frac{-u_{i}^{2}}{2 s_{k}^{2}}\right)
$$

and

$$
\alpha\left(u_{i}, u_{j}\right)=1+\exp \left(\frac{-\left(u_{i}-u_{j}+b\right)^{2}}{2 s_{k}^{2}}\right)-\exp \left(\frac{-b^{2}}{2 s_{a}^{2}}\right) .
$$

The exponential dependence of $k\left(u_{i}\right)$ on $u_{i}^{2}$ ensures that for extreme strategy values (both positive and negative) the fitness function will acquire large negative values. This means that strong deviations from the optimal strategy are evolutionarily strongly discouraged. The exponential dependendence of $\alpha\left(u_{i}, u_{j}\right)$ on $u_{i}-u_{j}$ ensures that strategies which are very different from each other will have the least competative effect, whereas the species will have a maximum competitive effect with itself. The parameter $b$ shifts the optimal result from $u_{i}-u_{j}=0$ so as to allow for a diversity of strategies. Illustrative examples of the above principles are given in Section 4 below.

\section{Strategy dynamics and homogeneous solutions}

In this section, we examine strategy dynamics in the context of reaction-diffusion equations. We then use this to examine the local convergence stability of ESS candidate solutions identified by the $G$-function approach of the previous section and illustrate the use of these equations with the help of numerical solutions for the Monod model system.

\subsection{Problem statement}

We consider a community of $r$ species, living in a one, two, or three dimensional space. We define a biological species as a collection of evolutionarily identical individuals that possess nearly 
identical strategies [58, 12]. We consider $N$ phenotypes for each species, each of which possesses a scalar strategy. In this system, all offspring inherit the same phenotype from a parent. For phenotype $j$ of species $i$ (phenotype $i j$ henceforth), we denote the constant strategy by $u_{i j}$ and the population density at location $\mathbf{x}$ and time $t$ as $z_{i j}(\mathbf{x}, t)$. The population dynamics of phenotype $i j$ is given by a reaction-diffusion equation similar to (2.1), which as before accounts for point-wise reactions (i.e., birth and death) along with migration by diffusion. For simplicity, we assume that diffusion coefficients vary with species, but not with phenotype. We also assume constant diffusion coefficients. Let:

$$
\begin{aligned}
\tilde{\mathbf{u}} & =\left[u_{11}, \ldots u_{1 N_{1}}, \ldots, u_{r 1}, . . u_{r N_{r}}\right] \\
\tilde{\mathbf{z}} & =\left[z_{11}, \ldots z_{1 N_{1}}, \ldots, z_{r 1}, \ldots z_{r N_{r}}\right], \\
\tilde{\mathbf{H}} & =\left[H_{11}, \ldots H_{1 N_{1}}, \ldots, H_{r 1}, \ldots H_{r N_{r}}\right], \\
\mathbf{D} & =\operatorname{diag}\left[d_{1}, \ldots, d_{r}\right] .
\end{aligned}
$$

where $H_{i j}$ is a fitness function of phenotype $i j$ and $d_{i}$ is the diffusion coefficient of the individuals of species $i$. The reaction-diffusion equation for each phenotype is then:

$$
\begin{aligned}
\partial_{t} z_{i j}(\mathbf{x}, t) & =H_{i j}(\tilde{\mathbf{u}}, \tilde{\mathbf{z}}(\mathbf{x}, t)) z_{i j}+d_{i} \Delta z_{i j}(\mathbf{x}, t) \\
i & =1 \ldots r, \quad j=1 \ldots N_{i}, \quad z_{i j} \geq 0,
\end{aligned}
$$

We further assume the Neumann boundary condition for (3.1) [25]:

$$
\frac{\partial z_{i j}}{\partial n}(\mathbf{x}, t)=\mathbf{0}, \quad \text { on } \quad \partial \Omega \times[0, \infty) .
$$

Define $z_{i}$, the population density of species $i$, at a specific location as the sum of all phenotype population densities:

$$
z_{i}(\mathbf{x}, t)=\sum_{j=1}^{N_{i}} z_{i j}(\mathbf{x}, t),
$$

and $u_{i}$, the mean strategy of species $i$, at a specific location as a weighted mean of phenotype strategies:

$$
u_{i}(\mathbf{x}, t)=\sum_{j=1}^{N_{i}} q_{i j}(\mathbf{x}, t) u_{i j},
$$

where $q_{i j}$ is the phenotype frequency at a specific location, defined as:

$$
q_{i j}(\mathbf{x}, t)=\frac{z_{i j}(\mathbf{x}, t)}{z_{i}(\mathbf{x}, t)} .
$$

We assume throughout that $z_{i}>0$. Note that the present treatment generalizes our assumption from Section 2, where $u_{i}$ was a constant. Because $u_{i}$ as defined by (3.4) depends on relative population proportions, and because these populations change with space and time, the value of $u_{i}$ now depends on the exact position in space and moment in time.

We are interested in formulating equations that would describe the coupled dynamics of the total population, $z_{i}(\mathbf{x}, t)$, and the mean strategy, $u_{i}(\mathbf{x}, t)$, of each species without the need for considering phenotypes explicitly. 


\subsection{The per-phenotype $G$-function}

A per-phenotype fitness generating function ( $G$-function) can be defined as follows:

Definition 5. A function $\tilde{G}(v, \tilde{\mathbf{u}}, \tilde{\mathbf{z}}(\mathbf{x}, t))$ is a $G$-function for phenotype ij if for every $i=1 \ldots r$, $j=1 \ldots N_{i}$, it obeys the relation: $\left.\tilde{G}(v, \tilde{\mathbf{u}}, \tilde{\mathbf{z}}(\mathbf{x}, t))\right|_{v=u_{i j}}=H_{i j}(\tilde{\mathbf{u}}, \tilde{\mathbf{z}})$.

Definition 5 is a generalization of definition 4 , where $v$ is a virtual strategy. Replacing $v$ by the strategy $u_{i j}$ results in the fitness function for individuals of type $i j$. We note that one does not have to use the $G$-function approach to study strategy dynamics and a fitness-function based formalism can also be pursued $[2,1,14,23,22,21]$. In the following, we shall replace $H_{i j}(\tilde{\mathbf{u}}, \tilde{\mathbf{z}})$ with $\left.\tilde{G}(v, \tilde{\mathbf{u}}, \tilde{\mathbf{z}}(\mathbf{x}, t))\right|_{v=u_{i j}}$ throughout.

\subsection{Population dynamics}

First, let us derive the boundary conditions for $z_{i}$. Using (3.3) we obtain:

$$
\frac{\partial z_{i}(\mathbf{x}, t)}{\partial n}=\frac{\partial \sum_{j=1}^{N_{i}} z_{i j}(\mathbf{x}, t)}{\partial n}=\sum_{j=1}^{N_{i}} \frac{\partial}{\partial n}\left(z_{i j}(\mathbf{x}, t)\right)=0, \text { on } \partial \Omega \times[0, \infty),
$$

where $n$ is the normal vector on the boundary pointing outwards. Thus, the Neumann boundary conditions hold for $z_{i}(\mathbf{x}, t)$ as well as for $z_{i j}$. This must be because each species is enclosed by the same uncrossable boundary that prevents the flux of its phenotypes.

To determine the population dynamics on a species basis, we differentiate (3.3) with respect to time:

$$
\partial_{t} z_{i}(\mathbf{x}, t)=\sum_{j=1}^{N_{i}} \partial_{t} z_{i j}(\mathbf{x}, t)=\left.z_{i} \sum_{j=1}^{N_{i}} \frac{z_{i j}}{z_{i}} \tilde{G}(v, \tilde{\mathbf{u}}, \tilde{\mathbf{z}}(\mathbf{x}, t))\right|_{v=u_{i j}}+d_{i} \Delta\left(\sum_{j=1}^{N_{i}} z_{i j}(\mathbf{x}, t)\right) .
$$

We define the per-species mean of the $G$-function as (we omit the spatial coordinates for notational convenience):

$$
\left.\bar{G}_{i}(\tilde{\mathbf{u}}, \tilde{\mathbf{z}}) \triangleq \sum_{j=1}^{N_{i}} q_{i j} \tilde{G}(v, \tilde{\mathbf{u}}, \tilde{\mathbf{z}})\right|_{v=u_{i j}} .
$$

Using (3.8) and (3.5), (3.7) can be written as:

$$
\partial_{t} z_{i}(\mathbf{x}, t)=z_{i}(\mathbf{x}, t) \bar{G}_{i}(\tilde{\mathbf{u}}, \tilde{\mathbf{z}})+d_{i} \Delta z_{i}(\mathbf{x}, t) .
$$

For simplifying the mathematical analysis, we make the following assumptions. We follow Vincent et al. (1993) in assuming that the distribution of populations and strategies is symmetric about their respective mean values and that the variance of the strategy distribution is small, so that a first order Taylor expansion of $G$ around the mean value is sufficient [58]. We can then 
replace the per-species mean value of the per-phenotype $G$-function by the value of the regular (per-species) $G$-function calculated using the per-species mean population and strategy values, i.e.:

$$
\bar{G}_{i}(\tilde{\mathbf{u}}, \tilde{\mathbf{z}})=\left.G(v, \mathbf{u}(\mathbf{x}, t), \mathbf{z}(\mathbf{x}, t))\right|_{v=u_{i}},
$$

where

$$
\begin{aligned}
& \mathbf{u}=\left[u_{1}, \ldots, u_{r}\right] \\
& \mathbf{z}=\left[z_{1}, \ldots, z_{r}\right]
\end{aligned}
$$

Note that $\mathbf{u}$ and $\mathbf{z}$ are "short" (length $r$ ) vectors of mean strategies and populations, as opposed to the "long" per-phenotype strategy and population vectors $\tilde{\mathbf{u}}$ and $\tilde{\mathbf{z}}$. Eq. (3.9) then becomes:

$$
\partial_{t} z_{i}(\mathbf{x}, t)=\left.z_{i}(\mathbf{x}, t) G(v, \mathbf{u}(\mathbf{x}, t), \mathbf{z}(\mathbf{x}, t))\right|_{v=u_{i}}+d_{i} \Delta z_{i}(\mathbf{x}, t) .
$$

Eq. (3.11) together with the boundary condition (3.6) completely define the population dynamics.

Although derived from phenotype considerations, the result is independent of any explicit treatment of phenotypes. This is because if the variance is small one can replace the exact dynamics of the phenotypic distribution with an overall dynamics on a per-specis basis. Indeed, (3.11) and (3.6) are the same as (2.1) and (2.3) which we have used in Section 2 as the starting point of an analysis that did not consider intraspecies strategy variation.

\subsection{Strategy dynamics}

We start our discussion of strategy dynamics by deriving the appropriate boundary conditions for the strategy. Using (3.4) and (3.5), we obtain:

$$
\frac{\partial u_{i}(\mathbf{x}, t)}{\partial n}=\frac{\partial}{\partial n} \sum_{j=1}^{N_{i}} \frac{z_{i j}}{z_{i}} u_{i j}=\sum_{j=1}^{N_{i}} u_{i j} \frac{\frac{\partial z_{i j}(\mathbf{x}, t)}{\partial n} z_{i}-\frac{\partial z_{i}(\mathbf{x}, t)}{\partial n} z_{i j}}{z_{i}^{2}}, \quad \text { on } \quad \partial \Omega \times[0, \infty) .
$$

But in light of (3.2) and (3.6), this implies that:

$$
\frac{\partial u_{i}(\mathbf{x}, t)}{\partial n}=0, \quad \text { on } \quad \partial \Omega \times[0, \infty) .
$$

The biological meaning of this boundary condition is that strategies cannot cross the boundary becuase strategies diffuse through the diffusion of the organism and thus must follow the population boundary condition.

We now derive the equation describing the dynamics of each species' mean strategy, $u_{i}$. Differentiating equation (3.4) with respect to time and using (3.5) we have

$$
\partial_{t} u_{i}=\sum_{j=1}^{N_{i}} \frac{z_{i} \partial_{t} z_{i j}-z_{i j} \partial_{t} z_{i}}{z_{i}^{2}} u_{i j}
$$

Using (3.1),(3.5), and (3.11), in (3.14) we arrive at: 


$$
\partial_{t} u_{i}=\sum_{j=1}^{N_{i}} q_{i j} u_{i j}\left(\left.\tilde{G}(v, \tilde{\mathbf{u}}, \tilde{\mathbf{z}}(\mathbf{x}, t))\right|_{v=u_{i j}}-\left.G(v, \mathbf{u}(\mathbf{x}, t), \mathbf{z}(\mathbf{x}, t))\right|_{v=u_{i}}\right)+\frac{d_{i}}{z_{i}} \sum_{j=1}^{N_{i}}\left(\Delta z_{i j}-q_{i j} \Delta z_{i}\right) u_{i j} .
$$

For the sake of simplicity we denote the first term on the RHS of (3.15) by $\partial_{t} u_{i A}$ and the second by $\partial_{t} u_{i B}$.

A Taylor series expansion of $\tilde{G}$ about $u_{i}$ yields to first order:

$$
\left.\left.\tilde{G}(v, \tilde{\mathbf{u}}, \tilde{\mathbf{z}}(\mathbf{x}, t))\right|_{v=u_{i j}}=\left.G(v, \mathbf{u}(\mathbf{x}, t), \mathbf{z}(\mathbf{x}, t))\right|_{v=u_{i}}\right)+\left.\frac{\partial G(v, \mathbf{u}(\mathbf{x}, t), \mathbf{z}(\mathbf{x}, t))}{\partial v}\right|_{v=u_{i}} \delta u_{i j}
$$

where

$$
\delta u_{i j}=u_{i j}-u_{i}
$$

is the deviation of $u_{i j}$ from the mean strategy $u_{i}$ and, based on the same assumptions as before, both the mean of the per-phenotype $G$-function and the mean of its derivative with respect to the virtual strategy have been replaced by the respective values of the per-species $G$-function. Using (3.16) and (3.17), $\partial_{t} u_{i A}$ can be written as:

$$
\left.\partial_{t} u_{i A} \cong \frac{\partial G}{\partial v}\right|_{v=u_{i}} \sum_{j=1}^{N_{i}} \delta u_{i j} q_{i j} u_{i j}=\left.\frac{\partial G}{\partial v}\right|_{v=u_{i}}\left(u_{i} \sum_{j=1}^{N_{i}} q_{i j} \delta u_{i j}+\sum_{j=1}^{N_{i}} q_{i j}\left(\delta u_{i j}\right)^{2}\right) .
$$

If we again assume that the distribution of populations and strategies is symmetric about their respective mean values, then $q_{i j}$ is symmetric about the mean whereas $\delta u_{i j}$ is antisymmetric about it. The first term on the RHS of equation (3.18) is therefore zero (see also Vincent et al. [58]). The second term is, by definition, the phenotypic variance of strategy values around the species' mean.

$$
s_{i}=\sum_{j=1}^{N_{i}} q_{i j}\left(\delta u_{i j}\right)^{2} .
$$

$s_{i}$ has been shown to be a constant of the dynamics [58] for the point process. In the Appendix, we show that this is also the case here, provided that the phenotypic variance has no spatial profile, i.e., it has the same value across all space. The latter assumption is biologically reasonable, because the phenotypic variance is related to genetic and epigenetic mutation rates and within the scope of our model there is no reason why those should be position-dependent. Thus:

$$
\partial_{t} u_{i A}=\left.\frac{\partial G}{\partial v}\right|_{v=u_{i}} s_{i}
$$

Turning to $\partial t u_{i B}$, using (3.4) and (3.5) with (3.15) yields, after some algebra:

$$
\partial_{t} u_{i B}=\frac{d_{i}}{z_{i}}\left[\Delta\left(z_{i} u_{i}\right)-u_{i} \Delta z_{i}\right]
$$


But:

$$
\Delta\left(z_{i} u_{i}\right)=u_{i} \Delta z_{i}+2 \nabla u_{i} \cdot \nabla z_{i}+z_{i} \Delta u_{i},
$$

where, as usual $\nabla$ is the gradient; i.e.,

so that:

$$
\nabla=\left(\frac{\partial}{\partial x_{1}}, \frac{\partial}{\partial x_{2}}, \frac{\partial}{\partial x_{3}}\right)
$$

$$
\partial_{t} u_{i B}=d_{i}\left[\Delta u_{i}+\frac{2 \nabla u_{i} \cdot \nabla z_{i}}{z_{i}}\right]
$$

Naturally, for species that decline and go to extinction, arrival at a steady state requires that the numerator in $\partial_{t} u_{i B}$ will go to zero faster than its denominator. This is indeed the case in the numerical solutions shown in Section 4.

Combining $\partial_{t} u_{i A}((3.18))$ with $\left.\partial_{t} u_{i B}(3.21)\right)$ yields our final result:

$$
\partial_{t} u_{i}(\mathbf{x}, t)=\left.s_{i} \frac{\partial G}{\partial v}\right|_{v=u_{i}}+d_{i}\left[\Delta u_{i}(\mathbf{x}, t)+\frac{2 \nabla u_{i}(\mathbf{x}, t) \cdot \nabla z_{i}(\mathbf{x}, t)}{z_{i}(\mathbf{x}, t)}\right] .
$$

Eq. (3.24) is similar to a reaction-diffusion equation. Just like (3.11), it has a point process term (which is the same as in the point process equation arrived at by Cohen et al. [12]) and a spatial term. The spatial term is comprised of a pure diffusion term and an additional term. Although the strategies are not self-diffusing, they do migrate via the diffusion of the populations. This explains why the strategy dynamics obey the same boundary conditions as the population density: if an animal cannot cross a boundary, neither can its strategy. However, because the strategies do not diffuse in the strict sense of the word, the spatial term of (3.24) is not simply the Laplacian.

Finally, (3.11) and (3.24) with their respective boundary conditions (3.6) and (3.13) define the dynamics of the system completely. These equations do not include explicitly any phenotypes. Evolutionary changes occur through the changes in phenotypic ratios. However, assuming a symmetric and small variance permits a quantitative discussion of the dynamics without explicit consideration of the phenotypes $i j$ and their frequencies $q_{i j}$.

\subsection{Convergence stability}

In this section, we apply the above population and strategy dynamics equations to test the local convergence stability of a candidate ESS corresponding to a spatially homogeneous equilibrium. We perform this stability analysis by studying the response of the system to small perturbations in the populations and strategies $\left(\delta z_{i}(\mathbf{x}, t)\right.$ and $\delta u_{i}(\mathbf{x}, t)$, respectively), followed by an analysis of the time-evolution of individual Fourier components of the perturbation. For simplicity, we denote equilibrium values by ' $*$ ' and avoid the explicit use of $(\mathbf{x}, t)$ and $(v, \mathbf{u}, \mathbf{z})$.

Applying a perturbation to Eqs. (3.11) and (3.24), we obtain:

$$
\begin{aligned}
\delta \partial_{t} z_{i} & =\delta\left(\left.z_{i} G\right|_{v=u_{i}^{*}, u^{*}, z^{*}}\right)+d_{i} \delta\left(\Delta z_{i}\right), \\
\delta \partial_{t} u_{i} & =s_{i} \delta\left(\left.\frac{\partial G}{\partial u_{j}}\right|_{v=u_{i}^{*}, u^{*}, z^{*}}\right)+d_{i} \delta\left(\Delta u_{i}\right)+2 d_{i} \delta\left(\frac{\nabla u_{i} \cdot \nabla z_{i}}{z_{i}}\right) .
\end{aligned}
$$


Evaluating the total differential of the various quantities in (3.25) yields:

$$
\begin{aligned}
\delta \partial_{t} z_{i}= & \left.G\right|_{v=u_{i}^{*}, u^{*}, z^{*}} \delta z_{i}+z_{i}^{*}\left[\left.\sum_{j=1}^{r} \frac{\partial G}{\partial z_{j}}\right|_{v=u_{i}^{*}, u^{*}, z^{*}} \delta z_{j}+\right. \\
& \left.\left.\frac{\partial G}{\partial v}\right|_{v=u_{i}^{*}, u^{*}, z^{*}} \delta u_{i}+\left.\sum_{j=1}^{r} \frac{\partial G}{\partial u_{j}}\right|_{v=u_{i}^{*}, u^{*}, z^{*}} \delta u_{j}\right]+d_{i} \Delta\left(\delta z_{i}\right), \\
\delta \partial_{t} u_{i}= & s_{i}\left[\left.\sum_{j=1}^{r} \frac{\partial^{2} G}{\partial v \partial z_{j}}\right|_{v=u_{i}^{*}, u^{*}, z^{*}} \delta z_{j}+\left.\sum_{j=1}^{r} \frac{\partial^{2} G}{\partial v \partial u_{j}}\right|_{v=u_{i}^{*}, u^{*}, z^{*}} \delta u_{j}+\right. \\
& \left.\left.\frac{\partial^{2} G}{\partial v^{2}}\right|_{v=u_{i}^{*}, u^{*}, z^{*}} \delta u_{i}\right]+\left.2 d_{i} \frac{\delta\left(\nabla u_{i} \cdot \nabla z_{i}\right) z_{i}-\delta z_{i}\left(\nabla u_{i} \cdot \nabla z_{i}\right)}{z_{i}^{2}}\right|_{u^{*}, z^{*}} \\
& +d_{i} \Delta\left(\delta u_{i}\right) .
\end{aligned}
$$

With some algebra, and noticing that at equilibrium $\nabla u_{i}=0$ and $\nabla z_{i}=0$, we can simplify (3.27) to:

$$
\begin{aligned}
\delta \partial_{t} u_{i}= & s_{i}\left[\left.\sum_{j=1}^{r} \frac{\partial^{2} G}{\partial v \partial z_{j}}\right|_{v=u_{i}^{*}, u^{*}, z^{*}} \delta z_{j}+\left.\frac{\partial^{2} G}{\partial v^{2}}\right|_{v=u_{i}^{*}, u^{*}, z^{*}} \delta u_{i}+\right. \\
& \left.\left.\frac{\partial^{2} G}{\partial v \partial u_{j}}\right|_{v=u_{i}^{*}, u^{*}, z^{*}} \delta u_{j}\right]+d_{i} \delta \Delta u_{i} .
\end{aligned}
$$

We expand the perturbations as a Fourier series over eigenfunctions of the Laplacian operator, namely:

$$
\delta z_{i}(\mathbf{x}, t)=\sum_{k} \delta b_{i_{k}}(t) \phi_{k}(\mathbf{x}) \quad \delta u_{i}(\mathbf{x}, t)=\sum_{k} \delta c_{i_{k}}(t) \phi_{k}(\mathbf{x})
$$

where $\phi_{k}(\mathbf{x})$ obeys:

$$
\Delta \phi_{k}(\mathbf{x})=-k^{2} \phi_{k}(\mathbf{x}), k=0,1, \ldots \infty .
$$

We substitute definition (3.29) in (3.26) and (3.28). After some algebra, and noting the generalized Fourier theorem [32], which states that two functions are equal if and only if all of their Fourier coefficients are equal, we obtain:

$$
\begin{aligned}
\delta \partial_{t} b_{i_{k}}(t)= & \left.G\right|_{v=u_{i}^{*}, u^{*}, z^{*}} \delta b_{i_{k}}(t)+z_{i}^{*}\left[\left.\sum_{j=1}^{r} \frac{\partial G}{\partial z_{j}}\right|_{v=u_{i}^{*}, u^{*}, z^{*}} \delta b_{j_{k}}(t)+\right. \\
& \left.\left.\frac{\partial G}{\partial v}\right|_{v=u_{i}^{*}, u^{*}, z^{*}} \delta c_{i_{k}}(t)+\left.\sum_{j=1}^{r} \frac{\partial G}{\partial u_{j}}\right|_{v=u_{i}^{*}, u^{*}, z^{*}} \delta c_{j_{k}}(t)\right]-k^{2} d_{i} \delta b_{i_{k}},
\end{aligned}
$$




$$
\begin{aligned}
\delta \partial_{t} c_{i_{k}}(t)= & s_{i}\left[\left.\sum_{j=1}^{r} \frac{\partial^{2} G}{\partial v \partial z_{j}}\right|_{v=u_{i}^{*}, u^{*}, z^{*}} \delta b_{j_{k}}(t)+\left.\frac{\partial^{2} G}{\partial v^{2}}\right|_{v=u_{i}^{*}, u^{*}, z^{*}} \delta c_{i_{k}}(t)\right. \\
& \left.+\left.\sum_{j=1}^{r} \frac{\partial^{2} G}{\partial v \partial u_{j}}\right|_{v=u_{i}^{*}, u^{*}, z^{*}} \delta c_{j_{k}}(t)\right]-k^{2} d_{i} \delta c_{i_{k}}(t) .
\end{aligned}
$$

Eqs. (3.30) and (3.31 can also be written in matrix form:

$$
\left[\begin{array}{r}
\delta \partial_{t} b_{1_{k}} \\
\vdots \\
\delta \partial_{t} b_{r_{k}} \\
\delta \partial_{t} c_{1_{k}} \\
\vdots \\
\delta \partial_{t} c_{r_{k}}
\end{array}\right]=(A)_{k}\left[\begin{array}{r}
\delta b_{1_{k}} \\
\vdots \\
\delta b_{r_{k}} \\
\delta c_{1_{k}} \\
\vdots \\
\delta c_{r_{k}}
\end{array}\right]
$$

where

$$
\begin{aligned}
& (A)_{k}=\left[\begin{array}{ll}
(A 1)_{k} & (A 2)_{k}
\end{array}\right] \text { and } \\
& (A 1)_{k}=\left[\begin{array}{ccc}
\left.z_{1} \frac{\partial G}{\partial z_{1}}\right|_{v=u_{1}^{*}, u^{*}, z^{*}}-k^{2} d_{1} & \cdots & \left.z_{1} \frac{\partial G}{\partial z_{r}}\right|_{v=u_{1}^{*}, u^{*}, z^{*}} \\
\vdots & \ddots & \vdots \\
\left.z_{r} \frac{\partial G}{\partial z_{1}}\right|_{v=u_{i}^{*}, u^{*}, z^{*}} & \cdots & z_{r} \frac{\partial G_{\sigma}}{\partial z_{\sigma}}-k^{2} d_{r} \\
\left.s_{1} \frac{\partial^{2} G}{\partial v \partial z_{1}}\right|_{v=u_{1}^{*}, u^{*}, z^{*}} & \cdots & \left.s_{1} \frac{\partial^{2} G}{\partial v \partial z_{r}}\right|_{v=u_{1}^{*}, u^{*}, z^{*}} \\
\vdots & \ddots & \vdots \\
\left.s_{r} \frac{\partial^{2} G}{\partial v \partial z_{1}}\right|_{v=u_{r}^{*}, u^{*}, z^{*}} & \cdots & \left.s_{r} \frac{\partial^{2} G}{\partial v \partial z_{r}}\right|_{v=u_{r}^{*}, u^{*}, z^{*}}
\end{array}\right] \\
& (A 2)_{k}=\left[\begin{array}{ccc}
z_{1}\left[\left.\frac{\partial G}{\partial u_{1}}\right|_{v=u_{1}^{*}, u^{*}, z^{*}}+\left.\frac{\partial G}{\partial v}\right|_{v=u_{1}^{*}, u^{*}, z^{*}}\right] & \cdots & \left.z_{1} \frac{\partial G}{\partial u_{r}}\right|_{v=u_{1}^{*}, u^{*}, z^{*}} \\
\vdots & \ddots & \vdots \\
\left.z_{r} \frac{\partial G}{\partial u_{1}}\right|_{v=u_{r}^{*}, u^{*}, z^{*}} & \cdots & z_{r}\left[\frac{\partial G_{r}}{\partial u_{r}}+\left.\frac{\partial G}{\partial v}\right|_{v=u_{1}^{*}, u^{*}, z^{*}}\right] \\
\left.s_{1} \frac{\partial^{2} G}{\partial v^{2}}\right|_{v=u_{1}^{*}, u^{*}, z^{*}}+\left.\frac{\partial^{2} G}{\partial v \partial u_{1}}\right|_{v=u_{1}^{*}, u^{*}, z^{*}}-k^{2} d_{1} & \cdots & \left.s_{1} \frac{\partial^{2} G}{\partial v \partial u_{r}}\right|_{v=u_{1}^{*}, u^{*}, z^{*}} \\
\vdots & \ddots & \vdots \\
\left.s_{r} \frac{\partial^{2} G}{\partial v \partial u_{1}}\right|_{v=u_{r}^{*}, u^{*}, z^{*}} & \cdots & s_{r}\left[\left.\frac{\partial^{2} G}{\partial v^{2}}\right|_{v=u_{r}^{*}, u^{*}, z^{*}}+\left.\frac{\partial^{2} G}{\partial v \partial u_{r}}\right|_{v=u_{r}^{*}, u^{*}, z^{*}}\right]
\end{array}\right]
\end{aligned}
$$

All the derivatives of $G$ in (3.33) are taken at $v=u_{i}^{*}, u_{i}=u_{i}^{*}$, and $z_{i}=z_{i}^{*}$.

Importantly, the stability of $(A)_{k}$ must be established for candidate ESS identified by the maximum principle. This ensures convergence stability rather than just uninvadibility (i.e., it is then consistent with the broad ESS definition 3 above). This may be stated as follows: 
Conclusion 1. A homogeneous spatial ecological equilibrium is locally stable to perturbation in the population density vector and the strategy vector if the real parts of the eigenvalues of all $(\mathbf{A})_{k}$ are negative.

The following two conclusions discuss what we can deduce from the convergence stability (CS) property of the point process on the CS of the spatially homogeneous equilibrium.

Conclusion 2. If an ESS has been found for a one-species point process, it is also an ESS strategy, in the sense that it is also CS for the spatially homogeneous equilibrium.

The uninvadibility has already been discussed in the previous section. As for convergence stability, following Proposition 1, the real part of the eigenvalue set of $\left|(A)_{k}-\lambda I\right|=0$ for the point process is negative. By adding the term $-k^{2} d$ where $d$ is the same for both entries, on the diagonal of the $2 \times 2(A)_{k}$ matrix, we get a new matrix $(B)_{k}$. The eigenvalues of $(B)_{k}$ are the set $\mu_{i}=\lambda_{i}-k^{2} d$. Since $k^{2} d$ is always $\geq 0$, the real parts of the eigenvalues $\mu_{i}$ are also negative.

A similar explanation holds for an $r \times r$ matrix where all species have the same diffusion coefficients. However, this case is not biologically likely.

An immediate result which stems from Conclusion 1 is

Conclusion 3. If an ESS has been found for the point process competition model with one species, then increasing the diffusion term $d_{i}$ or increasing $k^{2}$ can only accelerate the return to a convergent stable strategy spatially homogeneous equilibrium. From Conclusion 1, it is clear that increasing $d_{i}$ or $k^{2}$ will only make the eigenvalues of $(A)_{k}$ more negative, thereby accelerating the return to a spatially homogeneous equilibrium.

For more than one species, with more than one diffusion coefficient value, the matrix $(A)_{k}$ involves different $k^{2} d_{i}$ terms. In this case, eigenvalues with a positive real part may be created (depending on the form of the fitness function). The spatially homogeneous solution may therefore become unstable $[51,39,10]$ and the new equilibrium solution is no longer spatially homogeneous. If this is the case, spatial patterns may occur. This is considered further in Section 4.

\subsection{Example: the Monod competition model}

We illustrate the results developed in Section 3 using the Monod competition model for which, following Cohen et al. [12]:

$$
G(v, \mathbf{u}, \mathbf{z})=K-\frac{v^{2}}{a}+\frac{b \sum_{j=1}^{r} v u_{j} z_{j}}{\sum_{j=1}^{r} z_{j}}-\sum_{j=1}^{r} z_{j} .
$$

The corresponding fitness function is therefore:

$$
H_{i}(\mathbf{u}, \mathbf{z})=K-\frac{u_{i}^{2}}{a}+\frac{b \sum_{j=1}^{r} u_{i} u_{j} z_{j}}{\sum_{j=1}^{r} z_{j}}-\sum_{i=1}^{r} z_{i} .
$$

For a coalition of one, $u_{1}^{*}=0$ satisfies: 


$$
\left.\frac{\partial G}{\partial v}\right|_{v=u_{1}}=0 ;\left.\quad \frac{\partial^{2} G}{\partial v^{2}}\right|_{v=u_{1}}=\frac{-2}{a}
$$

for $a>0$ suggests an ESS. For this strategy, $z_{1}^{*}=K$ (the carrying capacity) and we obtain $\left.\frac{\partial^{2} G}{\partial v \partial u_{1}}\right|_{v=u_{1}}=b$. Using (3.33), $(A)_{k}$ is then:

$$
(A)_{k}=\left[\begin{array}{rr}
\left.z_{1} \frac{\partial G}{\partial z_{1}}\right|_{v=u_{1}^{*}, u^{*}, z^{*}}-k^{2} d_{1} & \left.z_{1} \frac{\partial G}{\partial u_{1}}\right|_{v=u_{1}^{*}, u^{*}, z^{*}} \\
\left.s_{1} \frac{\partial^{2} G}{\partial v \partial z_{1}}\right|_{v=u_{1}^{*}, u^{*}, z^{*}} & s_{1}\left[\left.\frac{\partial^{2} G}{\partial v^{2}}\right|_{v=u_{1}^{*}, u^{*}, z^{*}}+\left.\frac{\partial^{2} G}{\partial v \partial u_{1}}\right|_{v=u_{1}^{*}, u^{*}, z^{*}}\right]-k^{2} d_{1}
\end{array}\right] .
$$

For the present Monod problem this reduces to:

$$
(A)_{k}=\left[\begin{array}{rr}
-K-k^{2} d_{1} & 0 \\
0 & s_{1}\left(-\frac{2}{a}+b\right)-k^{2} d_{1}
\end{array}\right],
$$

and the eigenvalues are trivially $\lambda_{1}=-K-k^{2} d_{1}$ and $\lambda_{2}=s_{1}\left(-\frac{2}{a}+b\right)-k^{2} d_{1}$. Because the carrying capacity, $K$, and the variance, $s_{1}$, are always positive, the stability requirement for $k=0$ is simply

$$
-\frac{2}{a}+b<0
$$

In the examples below, we used different values of $a, b$, and $d_{1}$ to represent various scenarios of stability or instability. All numercial solutions were performed using Matlab.

Example 1. A strategy which is resistant to invasion but not convergent stable displays an instability with respect to a spatial perturbation.

With $a=1$ and $b=10$, requirement (3.35) is not fulfilled and $(A)_{0}$ is unstable, so that the same strategy as before is now not an invasion resistant strategy for the point process. With $k=1$ and $d_{1}=0.1,(A)_{1}$ is also unstable - its eigenvalues are $\lambda_{1}=-10.1, \lambda_{2}=0.06>0$. Indeed, Figure 1 shows that upon a small spatial perturbation with $k=1$, the perturbation increases in magnitude and causes the strategy to diverge from its equilibrium value. The strategy is therefore non-CS for a spatial perturbation as well.

Example 2. A non-CS strategy may lead to spatial speciation.

In Conclusion 2 we stated that a ESS for the point process is also a ESS for the spatial case. Here we demonstrate a case in which a non-CS strategy for the point process is also unstable for the spatial case and leads to two different strategy and population density values. Cohen et al. [12] demonstrated a possible mechanism for point process speciation by getting out of a local minimum of the $G$-function, which is not an uninvadable strategy, we show a similar spatial phenomenon (for a detailed example see Ref. [31]). 


\section{Presumed ESS on inhomogeneous surfaces}

In this Section, we consider in detail an example where diffusion leads to the formation of a spatially inhomogeneous solution. We show that the $G$-function approach developed in Section 2 is not applicable to this case. However, use of the strategy dynamics method developed in Section 3 results in new equilibrium solutions that feature spatially inhomogeneous strategies. Furthermore, these solutions are possibly ESS because extensive numerical simulations never revealed a successful invasion.

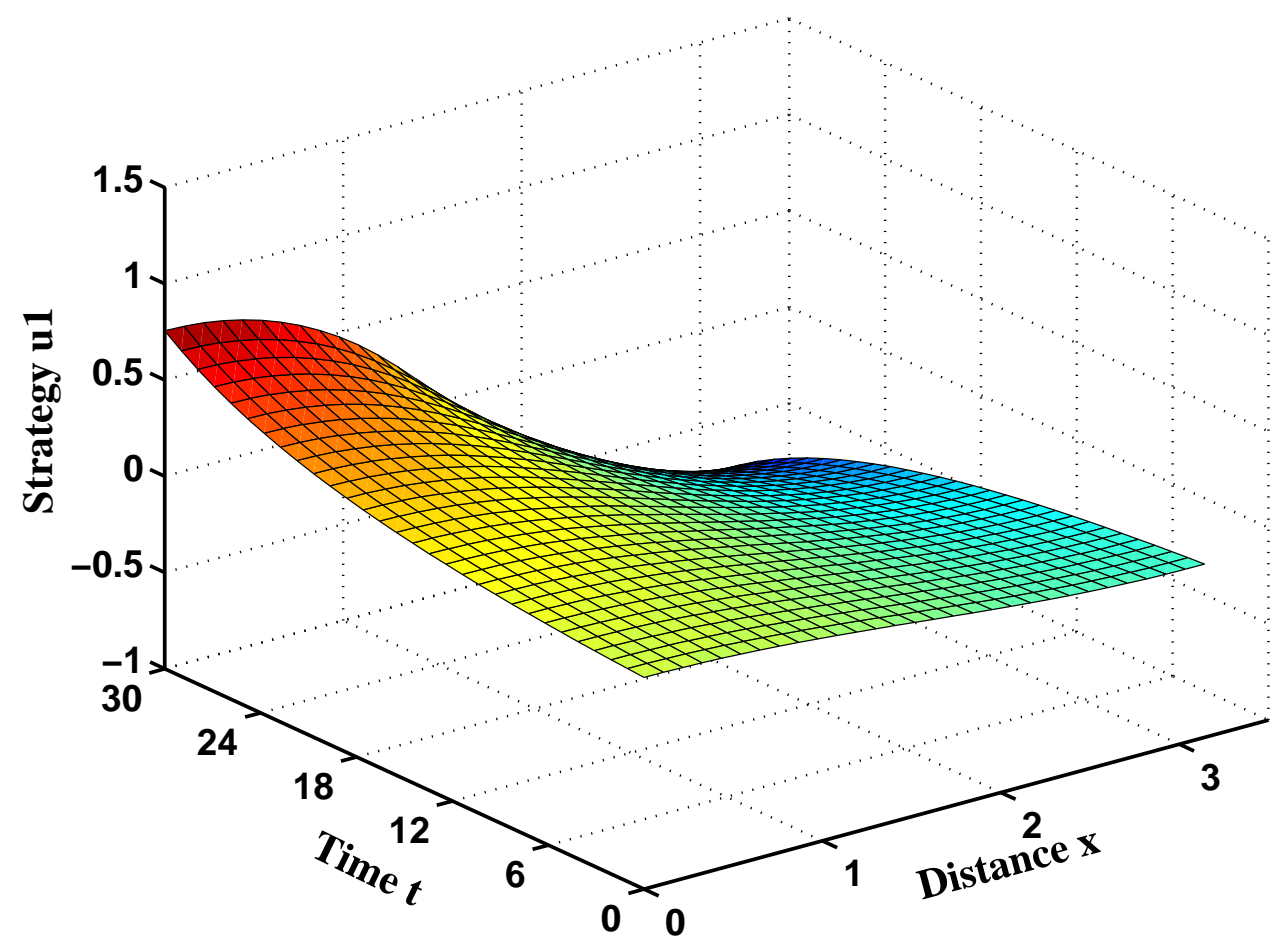

Figure 1: Dependence of the strategy value on time and space for one species possessing a point process strategy that is uninvadable but not CS in response to a spatial perturbation. The parameters are as in the previous example, except that $b=10, d_{1}=0.1$, and the initial perturbations are $\delta z_{1}(x, 0)=0.1 \cos (x), \delta u_{1}(x, 0)=0.1 \cos (x)$.

\subsection{Motivation and approach}

In Sections 2 and 3 we analyzed the spatial generalization of the $G$-function maximum principle including the corresponding strategy dynamics. Although the population and strategy dynamics given by (3.11), (3.24) and their associated boundary conditions (3.6), (3.13) are general, in the previous section we only studied explicitly equilibrium solutions that are spatially homogeneous for population sizes and strategy values alike. We now discuss the alternative: under which conditions does an inhomogeneous equilibrium arise, and what is its evolutionary meaning. 
In Section 2, Eq. (2.6) described the homogeneous locally stable solution. The $P_{E}$ solution assumed that at homogeneous equilibrium:

$$
\Delta \overline{\mathbf{z}}(\mathbf{x}, t)=\mathbf{0}
$$

and therefore:

$$
\overline{\mathbf{z}} \mathbf{H}(\mathbf{u}, \overline{\mathbf{z}}(\mathbf{x}, t), \mathbf{x}, t)=\mathbf{0} .
$$

We now relax this assumption by requiring only that the sum of (4.1) and (4.2) be zero. This invalidates the entire maximum principle analysis of Section 2 in general, and Proposition 1 and Conclusion 1 in particular. Therefore, we do not have a $G$-function maximum principle for the inhomogeneous spatial equilibrium. However, in the previous section we have shown how a solution of both the strategy dynamics and population dynamics leads us to the same ESS obtained from the maximum principle. Therefore, we use the explicit solution of the system, namely, $\mathbf{u}(\mathbf{x}, t), \mathbf{z}(\mathbf{x}, t)$, to study under which conditions an inhomogeneous solution is created and what it is.

Turing [51] was the first to point out the spatial pattern generating capability inherent in some reaction-diffusion systems. Until then the common thought was that diffusion has only a dampening effect. As we have pointed out at the end of Section 3, if the matrix $(A)_{k}$ in 3.33 involves different $k^{2} d_{i}$ terms, eigenvalues with a positive real part may be created and the spatially homogeneous solution may become unstable $[51,39,10]$ and may result in a spatial pattern. Such instability is termed diffusion-driven instability. In our attempts to produce such a situation for the present problem, our steps will be as follows: Because our interest is in evolutionary ecology, we use as an example a predator-prey model (which is equivallent to Turing's activator-inhibitor) presented by Segel and Jackson [46]. This model has been known to generate spatial patterns and so the proper diffusion coefficients that invoke Turing instability are known. We then make the various coefficients strategy dependent, thus turning this ecological model into an evolutionary game. Finally, we state the $G$-functions corresponding to the predator-prey rate equations and the proper strategy dynamics equations. Although the spatial Maximum Principle predicts a certain ESS, when we apply the destabilizing diffusion coefficients and perturb the system, we show that neither the populations nor the strategies converge to their homogeneous equilibrium values.

\subsection{The basic model}

The basic reaction-diffusion model used by Segel and Jackson [46] is:

$$
\begin{aligned}
& \frac{\partial C_{1}}{\partial t}=R_{1}\left(C_{1}, C_{2}\right)+d_{1} \Delta C_{1}, \\
& \frac{\partial C_{2}}{\partial t}=R_{2}\left(C_{1}, C_{2}\right)+d_{2} \Delta C_{2},
\end{aligned}
$$

where $C_{i}$ can be population density, chemical concentration, etc. and $R_{i}$ is some reaction term: We set:

$$
a_{i j}=\left.\frac{\partial R_{i}}{\partial C_{j}}\right|_{*},
$$




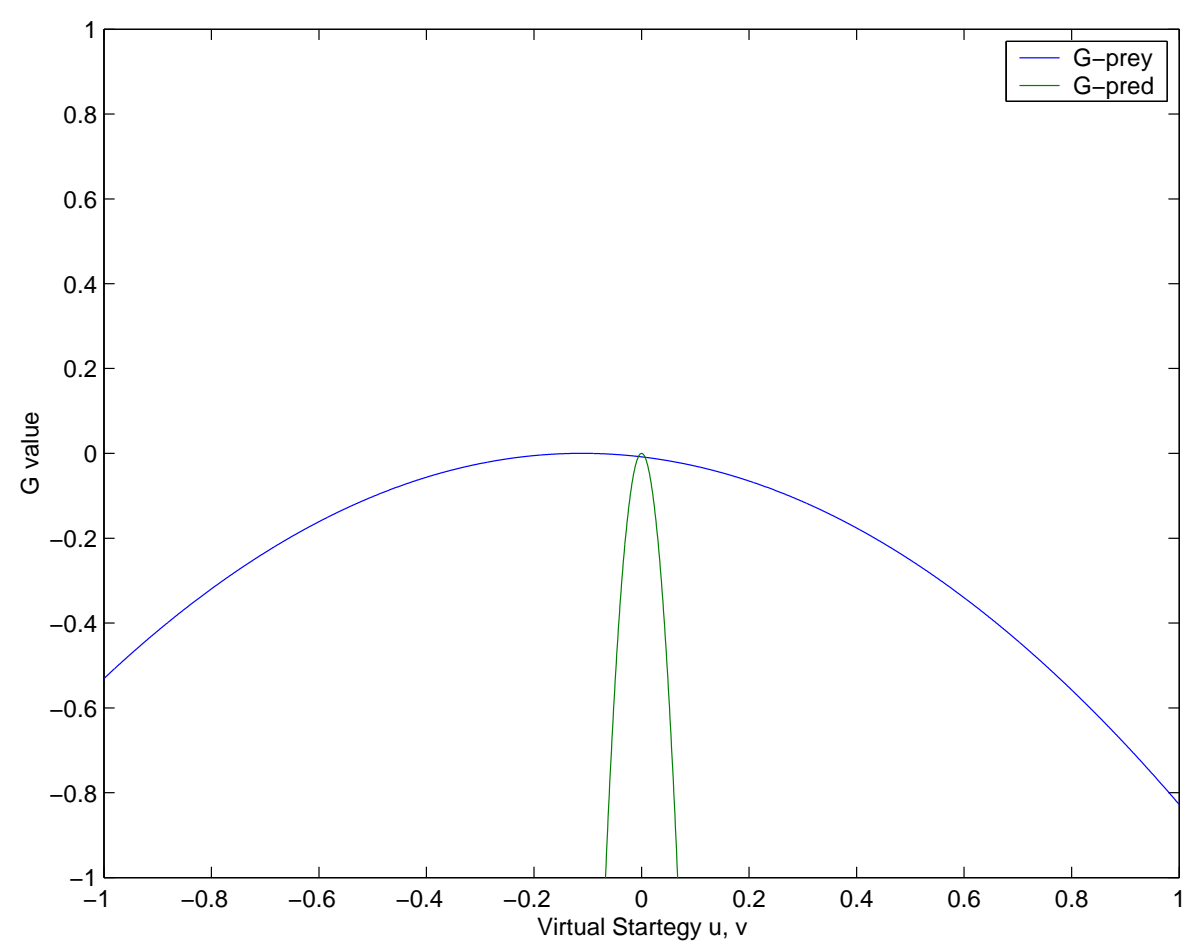

Figure 2: $G$-functions for the spatially homogeneous victim-exploiter model. Parameters are given in the text.

where '*' denotes a homogeneous equilibrium solution.

An unstable solution occurs when the stability matrix corresponding to (4.3) has at least one eigenfuction with a positive real-part. It has been shown [46] that sufficient conditions for stability of the point process are:

$$
\begin{aligned}
a_{11}+a_{22} & <0 \\
a_{11} a_{22}-a_{12} a_{21} & >0 .
\end{aligned}
$$

However, if the diffusion coefficient values obey

$$
d_{1} a_{22}+d_{2} a_{11} \geqslant 2 \sqrt{\left(d_{1} d_{2}\right)\left(a_{11} a_{22}-a_{12} a_{21}\right)}
$$

instability of the spatially homogeneous equlibrium occurs. The onset of instability is when (4.4) is an equality. These diffusion values will produce instability within a range of wave numbers (see Eq. (2.11)). When such instablity occurs, the minimal wave number for instability is given by:

$$
k_{\text {min }}^{2}=\left(d_{1} a_{22}+d_{2} a_{11}\right) / 2 d_{1} d_{2}
$$



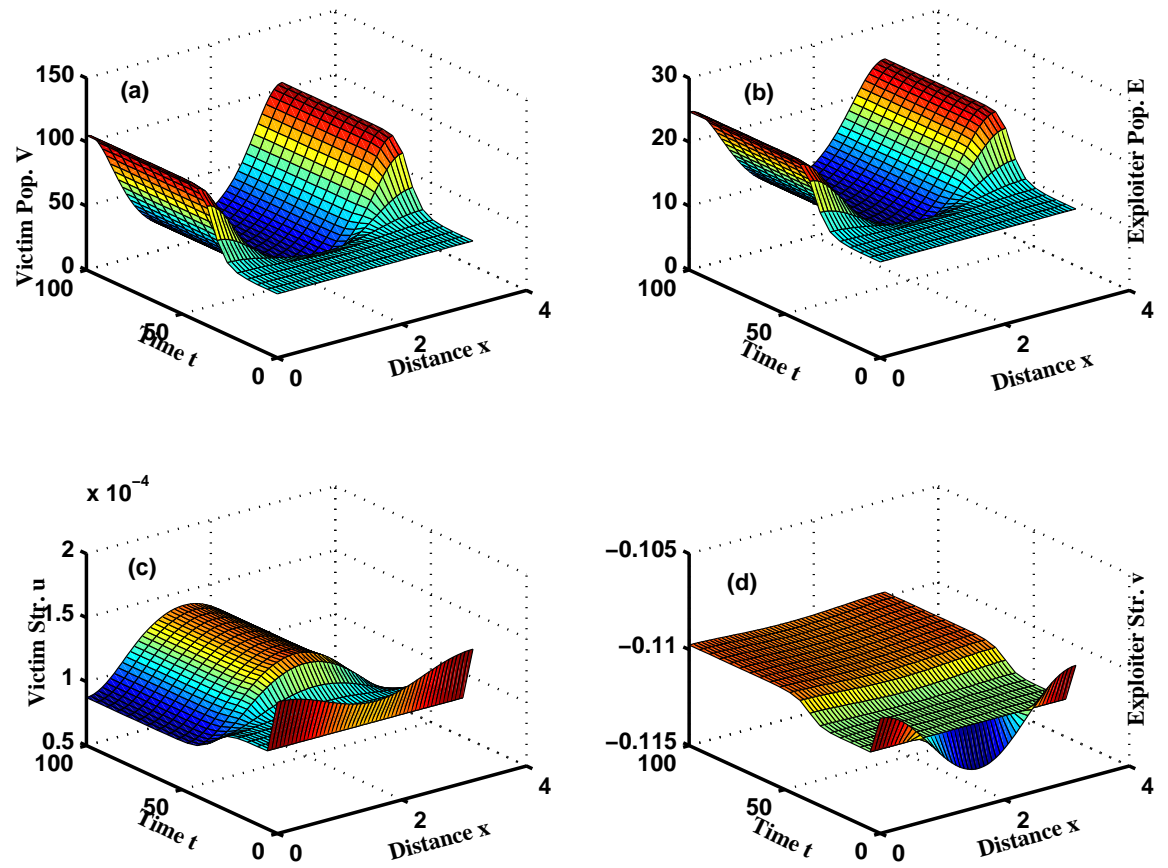

Figure 3: Time dependence of victim and exploiter population densities and strategies for two values of diffusion coefficients. Parameters are: Victim - $d_{1}=0.21, \delta V(x, 0)=50+0.01 \cos (2 x)$, $\delta u=1.4265 \cdot 10^{-4}+0.1 \cdot 10^{-4} \cos (2 x)$. Exploiter $-d_{2}=1, \delta E(x, 0)=15+0.003 \cos (2 x)$, $\delta v=-0.111+0.002 \cos (2 x)$. (a) Victim population density ; (b) Expliter population density ; (c) Victim startegy ; (d) Exploiter strategy.

\subsection{The exploiter-victim model}

Segel and Jackson [46] preferred naming their model exploiter-victim (rather than a predator-prey) model and we follow their notation. In (4.3), we substitute:

$$
\begin{aligned}
& C_{1}=V, \\
& C_{2}=E, \\
& R_{1}=V\left(\kappa_{0}+\kappa_{1} V\right)-a V E, \\
& R_{2}=b V E-c E^{2}
\end{aligned}
$$

to obtain:

$$
\begin{aligned}
& \frac{\partial V}{\partial t}=V\left(\kappa_{0}+\kappa_{1} V\right)-a V E+d_{1} \Delta V, \\
& \frac{\partial E}{\partial t}=b V E-c E^{2}+d_{2} \Delta E
\end{aligned}
$$

where $V$ is the victim population, $E$ is the exploiter population, $\kappa_{0}$ is the natural reproduction rate of the victim, $\kappa_{1}$ is the cooperativity constant among the victim, $a$ is the predation rate of victim 
by the exploiter, $b$ is the rate of conversion of preyed victim into exploiter offspring and $c$ is the exploiter mortality (attributed primarily to combat among exploiters).

We set the parameters to be:

$$
\begin{aligned}
\kappa_{0} & =1, \\
\kappa_{1} & =0.07, \\
a & =0.3, \\
b & =0.1, \\
c & =0.3333 .
\end{aligned}
$$

For the above parameters the spatially homogeneous equilibrium solution is:

$$
\begin{aligned}
& V^{*}=50, \\
& E^{*}=15 .
\end{aligned}
$$

Condition (4.4) predicts that under the above parameters (4.7) the critical diffusion ratio for spatial pattern formation is:

$$
\frac{d_{1}}{d_{2}} \tilde{=} 0.25 .
$$

Above this ratio no pattern will be formed and the homogeneous steady state will persist, and below it for $k_{\min }=2$ an inhomogeneous pattern should ensue.

\subsection{Game elements}

Now that we set the relevant parameters (4.7) and calculated the critical diffusion ratio (4.8), we can turn the coupled equations (4.6) into an evolutionary game by making the coefficients of (4.6) strategy dependent (as done by Brown and Vincent [9], for another ecological model). We impliclty use the multi $G$-function formalism (see Appendix 2 of Ref. [31]), however, for notational ease we use the respective fitness functions:

$$
\begin{aligned}
\partial_{t} V_{i} & =\kappa_{0}\left(u_{i}\right) V_{i}+\sum_{j} \kappa_{1}\left(u_{i}, u_{j}\right) V_{i} V_{j}-\sum_{j} a\left(u_{i}, w_{j}\right) V_{i} E_{j}+d_{i} \Delta V_{i}, \\
\partial_{t} E_{j} & =\sum_{i} b\left(u_{i}, w_{j}\right) V_{i} E_{j}-\sum_{i} c\left(w_{j}\right) E_{j} E_{i}+d_{j} \Delta E_{j},
\end{aligned}
$$



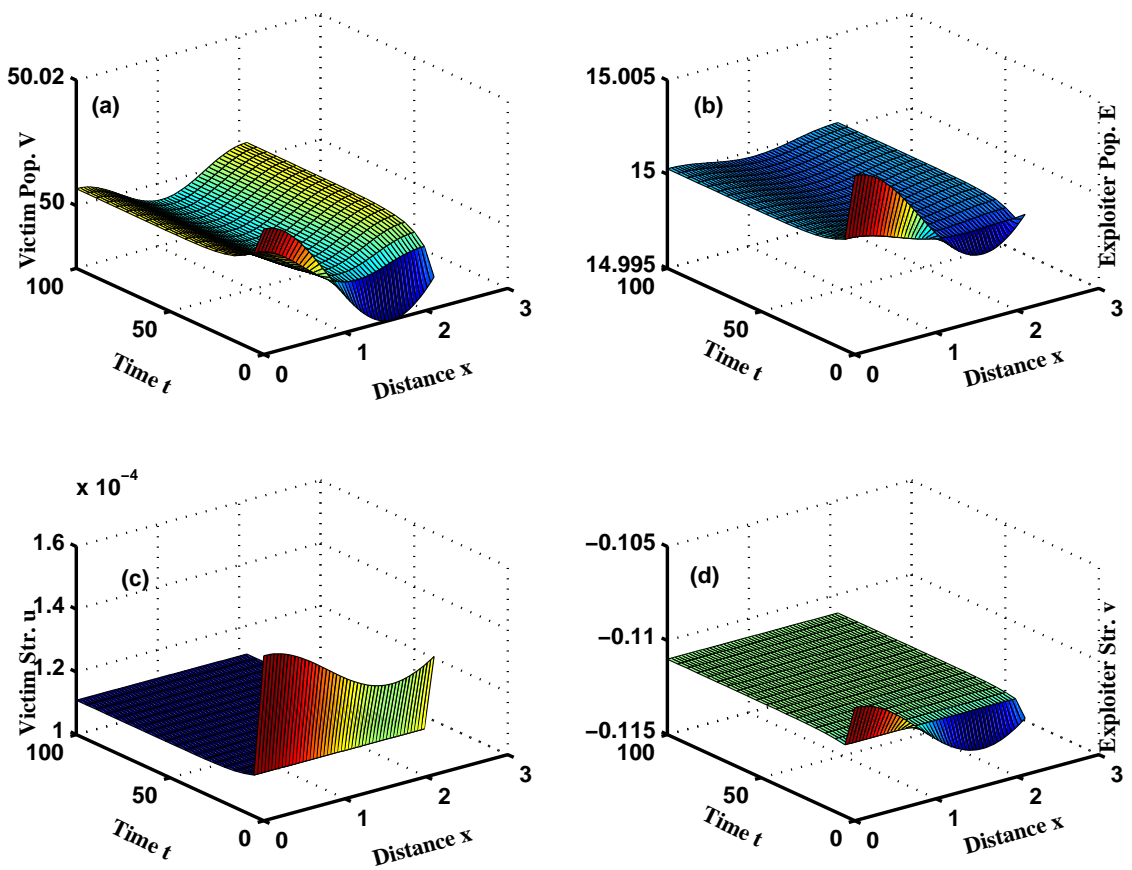

Figure 4: Time dependence of victim and exploiter population densities and strategies. All conditions are the same as in the previous figure, but the domain size is $2 / 3^{*}$ pi.

where:

$$
\begin{aligned}
\kappa_{0}\left(u_{i}\right) & =1 \exp \left[\frac{-u_{i}^{2}}{s_{k}^{2}}\right] / \exp \left[\frac{-u^{* 2}}{s_{k}^{2}}\right], \\
\kappa_{1}\left(u_{i}, u_{j}\right) & =0.07 \exp \left[\frac{-u_{i}^{2}}{s_{k}^{2}}\right] \exp \left[\frac{-\left(u_{i}-u_{j}\right)^{2}}{s_{C o}^{2}}\right] /\left(\exp \left[\frac{-u^{* 2}}{s_{k}^{2}}\right]\right), \\
a\left(u_{i}, w_{j}\right) & =0.3 \exp \left[\frac{-\left(u_{i}-w_{j}-0.1\right)^{2}}{s_{p}^{2}}\right] / \exp \left[\frac{-\left(u^{*}-w^{*}-0.1\right)^{2}}{s_{p}^{2}}\right], \\
b\left(u_{i}, w_{j}\right) & =0.1 \exp \left[\frac{-\left(u_{i}-w_{j}-0.1\right)^{2}}{s_{p}^{2}}\right] / \exp \left[\frac{-\left(u^{*}-w^{*}-0.1\right)^{2}}{s_{p}^{2}}\right], \\
c\left(w_{j}\right) & =0.3333 \exp \left[\frac{-w_{j}^{2}}{s_{c}^{2}}\right] / \exp \left[\frac{-w^{* 2}}{s_{c}^{2}}\right],
\end{aligned}
$$


with

$$
\begin{aligned}
s_{k} & =\sqrt{1}, \\
s_{C o} & =\sqrt{1}, \\
s_{p} & =\sqrt{100}, \\
s_{c} & =\sqrt{1000 a}, \\
u^{*} & =1.109877913429516 e-004, \\
w^{*} & =-0.11098779134295,
\end{aligned}
$$

and

$$
s=0.1
$$

where $u$ is the victim's strategy and $w$ is the exploiter's strategy. $s$ is the phenotypic strategy variance as in Eq. (3.19). $u^{*}, v^{*}$ are the spatially homogeneous ESS values of this game found through several iterations (usually 2 or 3 ) with some initial guess. The purpose of dividing by the exponents containing $u^{*}, v^{*}$ is to enforce a return to the original coefficients of (4.7) at ESS, thus obtaining the same $V^{*}, E^{*}$ values and consequently the same diffusion stability (or lack thereof).

By employing the above set of equations, we combine the strategy dependent parameter functions of Brown and Vincent (1992) with the ecological model of Segel and Jackson (1972). The functions of Brown and Vincent penalize strategy deviations from the ESS values and prevent the generation of extreme values. By dividing the exponential functions of Brown and Vincent by the value of the respective exponent at the ESS values (*), we ensure that when the system attains the homogeneous ESS values for both Victim and Exploiter, all parameters assume exactly the same values as in the Segel and Jackson (1972) model, thereby leading to their already known population desnsities.

To the best of our knowledge, this is the first attempt to test the applicability of the $G$-function determined point process ESS to spatial cases. Therefore, the main purpose of this example is actually to provide a counter-example, namely, to construct an example where the homogeneous ESS values are no longer relevant. Thus, no attempt at studying robustness with respect to the parameters was made.

Figure 2 shows the respective homogeneous $G$-functions obtained from the above model for the victim and exploiter. Clearly, the respective $u$ and $v$ values found for the homogeneous solution are indeed maximum values for the respective $G$-functions and are therefore what point-process $G$-function theory would predict to be ESS values.

We now write the corresponding strategy dynamics equations as presented in (3.24):

$$
\begin{aligned}
\frac{\partial u_{i}}{\partial t} & =s\left(\frac{\partial G_{V_{i}}}{\partial u_{i}}\right)+d_{i} \Delta u_{i}+2 d_{i} \frac{\nabla u_{i} \cdot \nabla V_{i}}{V_{i}} \\
\frac{\partial w_{j}}{\partial t} & =s\left(\frac{\partial G_{E_{i}}}{\partial w_{j}}\right)+d_{i} \Delta w_{j}+2 d_{j} \frac{\nabla w_{j} \cdot \nabla E_{j}}{E_{j}}
\end{aligned}
$$

In Section 3 we have shown that for the spatially homogeneous solution, the system strategy dynamics should lead it back to the original ESS values following an initial perturbation. 
Figure 3 shows the population density profiles and strategy profiles, respectively, obtained from the above model for two different diffusion coefficent ratios: one that should not induce instability of the homogeneous solution, and one that should. When the diffusion coefficient ratio is above the threshold level, no pattern appears and the equilibrium solution remains homogeneous for both populations and strategies.

However, when the system of Eqs. (4.9) and (4.12) is diffusion unstable, strategy dynamics do not lead it back to the original ESS values. Instead, a spatially inhomogeneous equilibrium solution develops for both the population sizes and for their strategies. This shows the inapplicability of the spatial, homogeneous maximum principle to the unstable system. This is because the spatial, homogeneous maximum principle predicts the homogeneous ESS to be locally stable, whereas here they were not convergent stable against spatial perturbation.

To illuminate the role of domain size on the steady state profile of species' strategy we show in Fig. 4(a)-(d) how a completely different profile of both populations and strategies is created when the domain size considered is changed to $2 / 3 *$ pi.

Even more profoundly, a point-process $G$-function based on the point-specific strategy value does not yield a meaningful analysis either. This is illustrated in Fig. 5, which shows the victim $G$-function as a function of the virtual strategy for the point-process equilibrium values and at three other instances in time (the last of which corresponds to the attainment of the spatially inhomogeneous equilibrium). Clearly, for any inhomogenous distribution the $G$-function's maximum value is not zero.

\subsection{Invasion scenarios: numerical solutions}

As mentioned in the introduction, the definition of an ESS (whether spatial or non-spatial) is that if the population adopts that strategy then no other strategy can co-exist with the ESS indefinitely. In the spatially homogeneous case, explicit use of the maximum principle or the use of strategy dynamics has led to the same prediction for the ESS. In the absence of a spatial maximum principle, are the spatially dependent strategies shown in Fig. 3 ESS? Here, we explore how the inhomogeneous system (4.9)-(4.12) reacts to an invader. Our simulations show that the prey or predator invader strategies we have entered into the predator-prey system meet one of two fates: either the strategy does not converge to that of the "host" prey and then its population declines until the invader is extinct, or the strategy converges to the profile of the inhomogeneous "host" prey and then the invader survives ("if you can't beat them, join them")(also see Ref. [31]). Interestingly, we found that the same two possible fates await the invading strategies tested when they compete in the corresponding homogeneous steady state system. This behavior of the inhomogeneous strategy "attractor" de facto leads us to believe that this spatial profile is indeed a candidate ESS for the spatially inhomogenous case. Note that we have performed this test under a scenario where the "invader" prey population was large - equal to that of the "host". Even so, the ESS value was restored, indicating its robustness.

We start the simulations of invasion scenarios with an example of the uninvadability for the non-spatial case in the absence of strategy dynamics, as discussed in Section 2. As an example, we study the case of an ESS coalition of two species possibly invaded by one mutant species (Example 


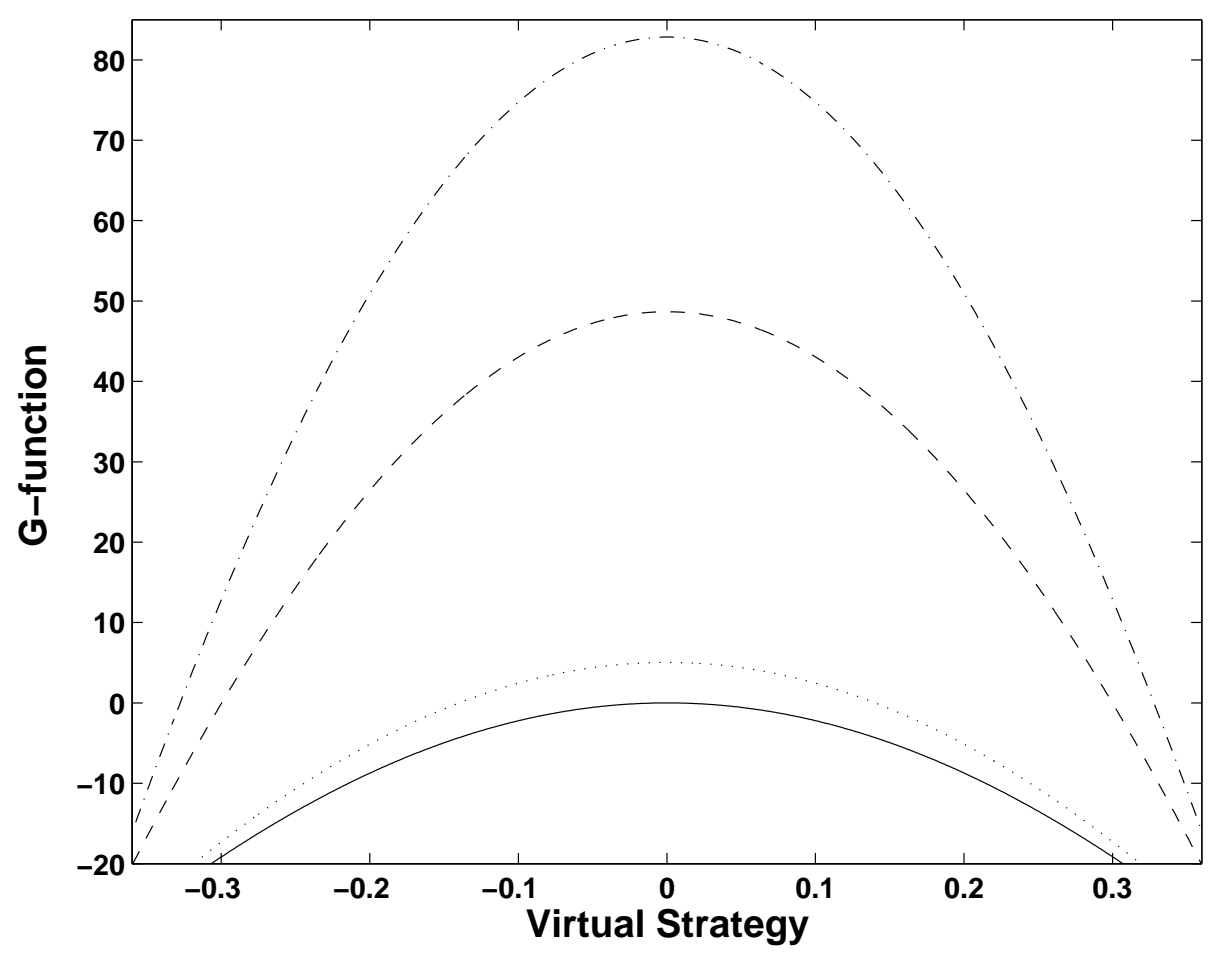

Figure 5: Victim $G$-functions for the same system studied in Fig. 3, at $x=p i / 25$, for the spatially homogeneous case (solid line) and for three different times: 25, 35, and 50 time units (dotted, dashed, and dash-dotted lines, respectively). At 50 time units, the system is at a spatially inhomogeneous equilibrium.

3). We use the following parameters:

$$
\begin{gathered}
k_{m}=100, \\
b=2, \\
s_{a}=2, \\
r=0.25,
\end{gathered}
$$

with $s_{k}=3.5$ for a two species ESS [59].

Example 3. Two species possessing a non-spatial ESS strategy, invaded by a mutant strategy

To find these strategies and population densities of a two species ESS coalition, we used criterion (2.13) and solved for $\mathbf{u}_{c}$ and $\overline{\mathbf{z}}$ using the method of Vincent et al. [59]. Solving the equations numerically using Mathematica ${ }^{T M}$, we found that $\mathbf{u}_{c}=[-0.29,3.08]$ and $\overline{\mathbf{z}}=[38.46,51.66]$. Fig. 6 shows the population density dynamics of a two-strategy coalition possessing the strategies that are ESS for the point process and for the homogeneous case, that is spatially perturbed from its equilibrium value $\overline{\mathbf{z}}$ and also invaded by a species with the non-ESS strategy of $u_{m}=5$. Given enough time, the non-spatial ESS strategies reach their respective locally stable, spatially homogeneous equilibria (Figs. 6a,b), while the population of the invader diminishes to zero (Fig. 6c). This 
indicates that, as in the case of a single-species ESS, $\overline{\mathbf{z}}$ is indeed an ESE for at least some domain of initial guesses, in agreement with Proposition 1.

In summary of this section, we have extended the point process ESS point-process maximum principle to a more general setting of a spatially homogeneous equilibrium solution. We have shown that for the latter case all point process ESS also hold for the spatial case and that the addition of a diffusion term can only hasten the decay of any spatial profile created by some initial perturbation. From an evolutionary ecology standpoint, this implies that for a species with population dynamics leading to a homogeneous population density, faster dispersal rates will lead to a faster return to equilibrium values.

\section{Concluding remarks}

Evolutionary games are a powerful tool for studying the impact of different species on each other's survival. As mentioned in the introduction, we have been aware for sometime now that human activities such as fragmentation of natural habitats (building roads, fences, and tunnels; settlements in wild areas; etc.) and introduction of exotic species have had a devastating effect on species biodiversity. Mathematically, this means that these activities change the composition of the surviving species in a habitat. The field of evolutionary games in general and the $G$-function approach in particular appear to be suitable tools for studying, and mathematically proving, the effect of spatial perturbation on the final composition of species in a habitat. In fact, such spatial effects even have analogies in other fields, e.g., wave turbulence [60]. In this work, we hope to have contributed to laying the foundations for a $G$-function approach to spatial evolutionary games. This method could be used not only to study the adverse affects of curent human spatial activities, but also to test spatial solutions to territorial problems, such as planning corridors for safe animal passage.

An important result of this study is that different rates of dispersal alone (and not just heterogeneous surroundings, as in, e.g., Ref. [7]) are sometimes enough to destabilize an otherwise evolutionarily stable system and especially to affect the type and profile of i evolutionarily viable strategies. To establish this, we used the reaction-diffusion equation as our basic model system. This is a relatively simple model and it describes quite accurately movement in chemicals and (at least) in simple life forms (e.g., bacteria, slime molds, etc.).

As with every model, our approach has limitations and assumptions. Most importantly, using the reaction-diffusion equation we assume that populations vary infinitesimally (as opposed to discretely), we allow for local interaction only, and we do not allow local stoachasticity [15]. In addition, the reaction-diffusion equation allows for several individuals to exist at the same location, whereas populating rules may exclude the existence of two competitors on the same site [16].

The reaction-diffusion approach is by no means the only way to include spatial effects. Other models, e.g., the patch model, allow for specific spetial arrangements and for differential accesibility to a patch. Understanding the underlying assumptions behind our approach is important because, as shown by Durret and Levin (1994), different models may yield different predictions. For example, Durret and Levin (1994) showed a case where a patch model predicted a two species solution whereas a reaction-diffusion model arrived at a one species solution. Recent work intro- 


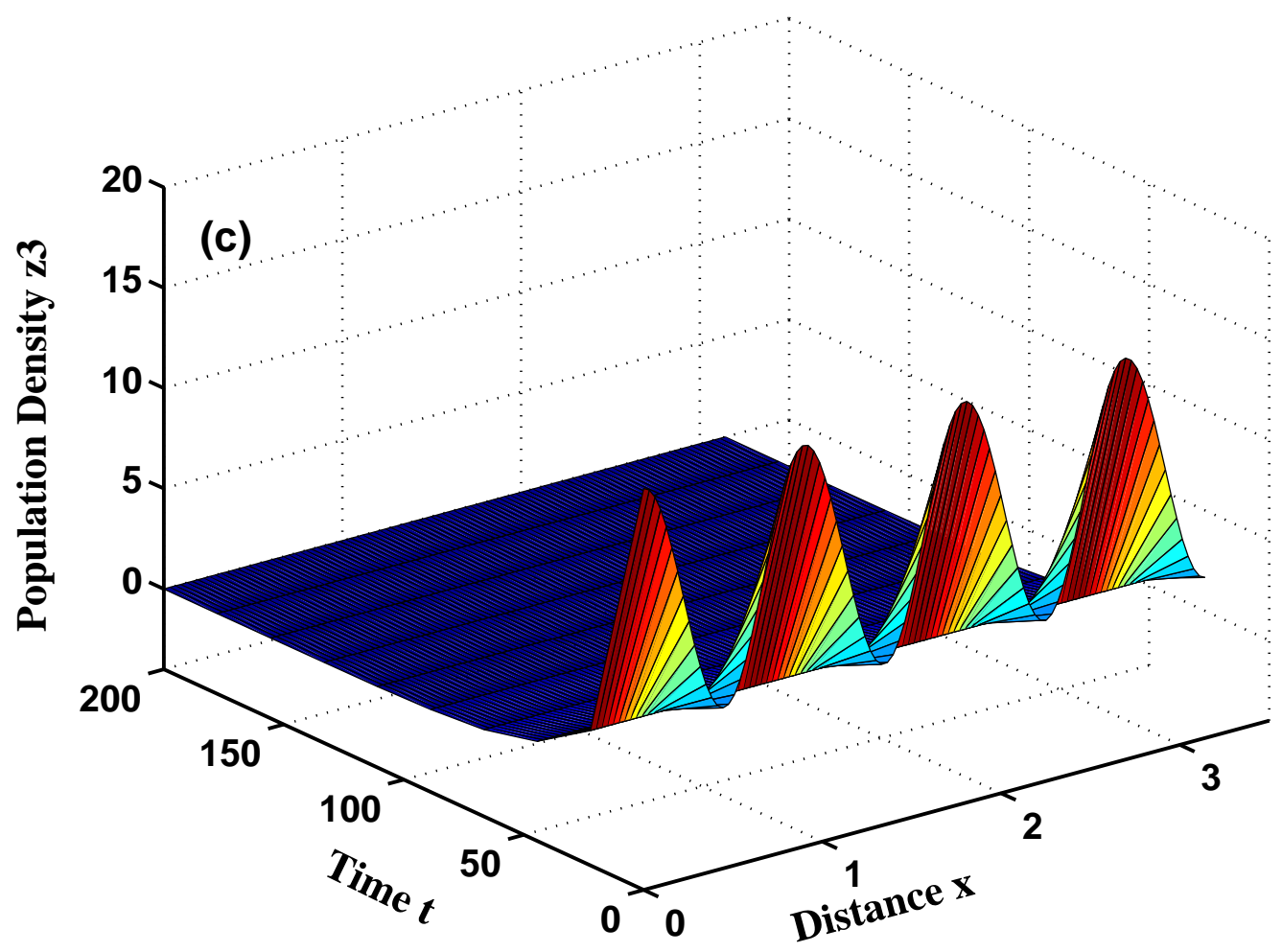

Figure 6: Dependence of the population density on time and space for two species possessing non-spatial ESS strategies and one species possessing a mutant strategy (a-c, respectively). $d_{1}=$ $d_{2}=d_{3}=1$ and the initial populations are: (a) $z_{1}(x, 0)=20+7 \cos (5 x)$, (b) $z_{2}(x, 0)=$ $80+8 \cos (3 x),(\mathrm{c}) z_{3}(x, 0)=10+6 \cos (7 x)$. 
duced space to the evolutionary process by focusing on the dynamics of metapopulations. There, the dynamics of dispersal strategy was studied [43, 38, 26]. This would be analogous to our diffusion coefficient. For example, Parvinen [43] analyzed the adaptive dynamics of a discrete-time metapopulation model and found requirements for an ESS to exist.

In the non-spatial system, the $G$-function maximum principle has proven to be - useful in finding candidates for an ESS [59]. Our next goal, therefore, was the generalization of the the $G$-function maximum principle to include spatial effects. Our generalization included also multiple $G$-function systems, which are useful for, e.g., studying predator-prey systems. The spatial $G$-function maximum principle was found to be meaningful for reaction diffusion sytems ending in a homogeneous steady-state. Yet we were still left without an ESS predictor for a inhomogeneous steady-state.

For the non-spatial case, strategy dynamics has proven to be a useful tool in "leading" the strategies to their ESS values. Cohen et al. [12] have shown the complete set of requirements for a point process ESS to exist. They also introduced convergence stability to the original definition of ESS by Maynard-Smith and Price [37]. These requirements included the stability of both the populations and their respective strategies. Convergence stability through a fitness function approach was also presented by Abrams and Matsuda [2]. Another accepted approach is the explicit use of selection of mutation models [14]. This approach has led to the discovery of shifting evolutionary isoclines and the slowing down of the evolutionary path). Another approach is the use of pairwise resident-invader contests $[23,22,21]$. It has lead to many interesting insights regarding evolutionary branching, evolutionarily singular strategies, and the "inheritance" of a resident's attractor by a mutant invader $[23,22,21]$. Although a natural evolutionary scenario may include pairwise competition, it is a special case of the more inclusive approach which takes into consideration the availability of all possible trait values in the game. We chose to use the fitness generating function, which does take this consideration.

For the case of the homogeneous, locally stable equilibrium, we proved that if an ESS exists for the point process, then for one species addition of diffusion in space will only accelerate the return to a spatially homogeneous equilibrium. We have also shown it is impossible to stabilize a non-CS univadable strategy by adding diffusion. The mechanism for speciation in a point process has been studied extensively in recent years $[23,22,12]$. We showed here that a $G$-function minimum which is not an ESS can lead to speciation into two different convergent stable strategies in a spatial context as well. Although we did not present here an example of an instability induced by a spatial perturbation in strategies for more than one species (akin to a Turing instability induced by a perturbation in populations [51]), this study does not preclude this possibility. This was probably not observed by us because the Lotka-Volterra competition model is not easily destabilized [3].

A popular model which is known to create patterns is the predator-prey model [46]. This model does not require spatial heterogeneity to cause heterogeneous equilibrium population. Instead, the heterogeneity is inherent in the system. To treat this model we used the spatial multi $G$-function approach and added game elements to the coefficients in the predator-prey model. Indeed, spatially inhomogeneous equilibirium populations and strategies arise in this case. In the absence of a spatial maximum principle that would constitute a direct proof of an ESS (or lack thereof), we tested the invasibilty of the obtained equilibrium strategy. Consistent with the behavior of the 
homogeneous system, the steady-state strategies held their ground against both prey and predator selected invading strategies. This leads us to the conclusion that although we lack a formal spatial maximum principle for the inhomogeneous case, we have perhaps provided an alternate tool of identifying ESS candidates.

As we have mentioned, our analysis dealt with scalar strategies. It would be interesting to generalize the approach to vector strategies as well. Another possibly important suggestion for further research stemming from this work is, therefore, to seek a generalization of the maximum principle that would be appropriate to a spatially inhomogeneous equilibrium solution.

\section{Acknowledgements}

This article is dedicated to Thomas L. Vincent, a pioneer of evolutionary game theory.

\section{References}

[1] P.A. Abrams. Adaptive dynamics: Neither F nor G. Evol. Ecol. Res., 3 (2001), 369-373.

[2] P.A. Abrams, H. Matsuda. Evolutionarily unstable fitness maxima and stable fitness minima of continuous traits. Evol. Ecol., 7 (1993), 465-487.

[3] D. Alonso, F. Bartumeus, J. Catalan. Mutual interference between predators can give rise to Turing spatial patterns. Ecology, 83 (2002), 28-34.

[4] H. Anton.and C. Rorres. Elementary linear algebra: applications version. 8th Edition. John Wiley \& Sons, New York, 2000.

[5] J. Apaloo. Revisting strategic models of evolution: The concept of neighborhood invader strategies. Theor. Pop. Biol.,52 (1997), 71-77.

[6] N.F. Britton. Reaction-diffusion equations and their applications to biology. Academic Press, New York, 1986.

[7] J.S. Brown, N.B. Pavlovic. Evolution in heterogeneous environments - effects of migtation on habitat specialization. Evol. Ecol. 6 (1992),360-382.

[8] J.S. Brown, T.L. Vincent. A theory for the evolutionary game. Theor. Pop. Biol., 31 (1987), 140-166.

[9] J.S. Brown, T.L. Vincent. Organiztion of predator-prey communities as an evolutionary game. Evolution, 46 (1992), 1269-1283.

[10] R.G. Casten, C.J. Holland. Stability properties of solutions of systems of reaction-diffusion equations. SIAM J. Appl. Math. 33 (1977), 353-364. 
[11] Y. Cohen, J. Pastor, T.L. Vincent. Evolutionary strategies and nutrient cycling in ecosystems. Evol. Ecol. Res., 2 (2000), 719-743.

[12] Y. Cohen, T.L. Vincent, J.S. Brown. A G-function approach to fitness minima, fitness maxima, evolutionarily stable strategies and adaptive landscapes. Evol. Ecol. Res., 1 (1999), 923-942.

[13] R. Cressman, G.T. Vickers. Spatial and density effects in evolutionary game theory. Math. Biol., 184 (1997), 359-369.

[14] U. Dieckmann, R. Law. The dynamical theory of coevolution: A derivation from stochastic ecological processes. J. Math. Biol., 34 (1996), 579-612.

[15] R. Durrett, S. Levin. The importance of being discrete (and spatial). Theor. Pop. Biol., 46 (1994), 363-394.

[16] R. Durrett, S. Levin. Allelopathy in spatially distributed populations. J. Theor. Biol., 185 (1997), 165-171.

[17] I. Eshel. Evolutionary and continuous stability. J. Theor. Biol. 108 (1983), 99-111.

[18] I. Eshel. On the changing concept of evolutionary population stability as a reflection of a changing point of view in the quantitative theory of evolution. J. Math. Biol. 34 (1996), 485510 .

[19] I. Eshel, U. Motro. Kin selection and strong evolutionary stability of mutual help. Theor. Pop. Biol. 19 (1981), 420-433.

[20] G. Gause. The struggle for existence. Williams and Wilkins, Baltimore, 1934.

[21] S.A.H. Geritz, M. Gyllenberg, F.J.A. Jacobs, K. Parvinen. Invasion dynamics and attractor inheritance. J. Math. Biol., 44 (2002), 548-560.

[22] S.A.H. Geritz, S.A.H. Kisdi, G. Meszéna, J.A.J. Metz. Evolutionary singular strategies and the adaptive growth and branching of the evolutionary tree. Evol. Ecol., 12 (1998), 35-57.

[23] S.A.H. Geritz, J.A.J. Metz. É. Kisdi, G. Meszéna. Dynamics of adaptation and evolutionary branching. Physical Review Letters 78, 2024-2027.

[24] A. Gorban. Selection theorem for systems with inheritance. Math. Model. Nat. Phenom., 2 (2007), 1-45.

[25] P. Grindrod. The theory and applications of reaction-diffusion equations: patterns and waves. 2nd Edition. Clarendon press, Oxford, 1996.

[26] M. Gyllenberg, J.A. Metz. On fitness in structured metapopulations. J. Math. Biol., 43 (2001), 545-560. 
[27] K.P. Hadeler. Diffusion in Fisher's population model. Rocky Mountain J. Math., 11 (1981), $39-45$.

[28] J. Haldane. The causes of evolution. Princeton University Press, 1932.

[29] W.G.S. Hines. Evolutionary stable strategies: A review of basic theory. Theor. Pop. Biol., 31 (1987), 195-272.

[30] V. C.L. Hutson, G.T. Vickers. Travelling waves and dominance of ESS's. J. Math. Biol., 30 (1992), 457-471.

[31] N. Kalev-Kronik. Evolutionary games in space. Ph.D. Thesis, University of Minneosta, 2006.

[32] W. Kaplan. Advanced calculus. Addison-Wesley, Reading, 1952.

[33] C.L. Lehman, D. Tilman. Spatial Ecology : The Role of Space in Population Dynamics and Interspecific Interactions, chapter: Competition in Spatial Habitats.. Princeton University Press, Princeton, 1997.

[34] J.L. Lions. Equations differentielles operationelles. Springer-Verlag, New-York, 1961.

[35] S. Lipschutz. Linear algebra. McGraw-Hill, New York, 1991.

[36] J. Maynard-Smith. Evolution and the theory of games. Cambridge University Press, Cambridge, 1982.

[37] J. Maynard-Smith, G. Price. The logic of animal conflict. Nature, 246 (1973), 15-18.

[38] J.A.J. Metz, M. Gyllenberg. How should we define fitness in structured metapopulation models?. Proc. Royal Soc. London B, 268 (2001), 499-508.

[39] J. Murray. Mathematical biology, 2nd Edition, Springer-Verlag, Berlin, 1993.

[40] C. Neuhauser. Habitat destruction and competitive coexistence in spatially explicit models with local interactions. J. Theor. Biol., 193 (1998), 445-463.

[41] C. Neuhauser, S.W. Pacala. An explicit spatial version of the lotka-volterra model with interspecific competition. Ann. Appl. Probab., 9 (1999), 1226-1259.

[42] H.G. Othmer, L.E. Scriven. Interactions of reaction and diffusion in open systems. Ind. Eng. Chem. Fund., 8 (1969), 302-313.

[43] K. Parvinen. Evolution of migration in a metapopulation. Bul. Math. Biol., 61 (1999), 531550.

[44] H. Qian, J. Murray. A simple method of parameter space determination for diffusion-driven instability with three species. Appl. Math. Let., 9 (2001), 405-411. 
[45] A. Sasaki, I. Kawaguchi, A. Yoshimori. Spatial mosaic and interfacial dynamics in a Müllerian mimicry system. Theor. Pop. Biol., 61 (2002), 49-71.

[46] L.E. Segel, J.L. Jackson. Dissipative structure: An explanation and an ecological example. J. Theor. Biol., 37 (1972), 545-559.

[47] J. Smoller. Shock waves and reaction-diffusion equations. Springer-Verlag, New York, 1983.

[48] T. Takada, J. Kigami. The dynamical attainability of ESS in evolutionary games. J. Math. Biol., 29 (1991), 513-529.

[49] P.D. Taylor. Evolutionary stability in one-parameter models under weak selection. Theor. Pop. Biol., 36 (1989), 125-143.

[50] D. Tilman, P. Kareiva eds. Spatial ecology : the role of space in population dynamics and interspecific interactions. Princeton University Press, Princeton, 1997.

[51] A.M. Turing. On the chemical basis of morphogenesis. Phil. Trans. B., 237 (1952), 37-37.

[52] G.T. Vickers, Spatial patterns and ESS's. J. Theo. Biol., 140 (1989), 129-135.

[53] G.T. Vickers,V.C.L. Hutson, C.J. Budd. Spatial patterns in population conflicts. J. Math. Biol., 31 (1993), 411-430.

[54] T. Vincent, Evolutionary games. J. Optim. Theor. Appl., 46 (1985), 605-612.

[55] T. Vincent, J. Brown. Evolution under nonequilibrium dynamics. Math. Model., 8 (1987), $766-771$.

[56] T.L. Vincent, J. Brown. Evolutionary game theory, natural selection, and Darwinian dynamics. Cambridge University Press, Cambridge, 2005.

[57] Vincent, T. Evolutionary stable strategies in differential and difference equation models. Evol. Ecol., 2, (1988), 321-337.

[58] T.L. Vincent, Y. Cohen, J.S. Brown. Evolution via strategy dynamics. Theor. Pop. Biol., 44 (1993), 149-176.

[59] T.L. Vincent, M.V. Van, G.S. Goh. Ecological stability, evolutionary stability, and the ESS Maximum Principle. Evol. Ecol., 10 (1996), 567-591.

[60] V. Zakharov, V.S. L'vov, S.S. Starobinets. Spin-wave turbulence beyond the parametric excitation threshold. Soviet Physics Uspekhii, 17 (1975), 896-919. 


\section{Appendix - Time dependence of the phenotypic variance}

In Eq. (3.19) of Section 3 we have defined the phenotypic variance as:

$$
s_{i}(\mathbf{x}, t)=\sum_{j=1}^{N_{i}} q_{i j}(\mathbf{x}, t)\left(\delta u_{i j}\right)^{2}
$$

To determine the time-dependence of the phenotypic variance, consider that its value at time $t+d t$, namely,

$$
s_{i}(\mathbf{x}, t+d t)=\sum_{j=1}^{N_{i}} q_{i j}(\mathbf{x}, t+d t)\left(\delta u_{i j}\right)^{2}
$$

is determined by the value of the phenotype frequency, $q_{i j}(\mathbf{x}, t+d t)$. With the help of Eq. (3.5), the latter quantity can be expressed as:

$$
q_{i j}(\mathbf{x}, t+d t)=\frac{z_{i j}(\mathbf{x}, t+d t)}{z_{i}(\mathbf{x}, t+d t)}=\frac{z_{i j}(\mathbf{x}, t)+\dot{z}_{i j}(\mathbf{x}, t) d t}{z_{i}(\mathbf{x}, t)+\dot{z}_{i}(\mathbf{x}, t) d t}
$$

Using Eqs. (3.1) and (3.11), we obtain:

$$
q_{i j}(\mathbf{x}, t+d t)=\frac{z_{i j}+\left(\widetilde{G} z_{i j}+d_{i} \nabla^{2} z_{i j}\right) d t}{z_{i}+\left(G z_{i}+d_{i} \nabla^{2} z_{i}\right) d t},
$$

where for simplicity of notation the arguments $(\mathbf{x}, t)$, as well as arguments of the $G$-function, have been omitted. Using Eq. (3.16), this can be further expressed as:

$$
\begin{aligned}
& q_{i j}(\mathbf{x}, t+d t)= \\
& =\frac{z_{i j}+\left(\left(G+\frac{\partial G}{\partial v} \delta u_{i j}\right) z_{i j}+d_{i} \nabla^{2} z_{i j}\right) d t}{z_{i}+\left(G+d_{i} \nabla^{2} z_{i}\right) d t} \\
& =\frac{z_{i j}\left[1+\left(G+d_{i} \frac{\nabla^{2} z_{i}}{z_{i}}\right) d t\right]+z_{i j}\left[\frac{\partial G}{\partial v} \delta u_{i j}+d_{i}\left(\frac{\nabla^{2} z_{i j}}{z_{i j}}-\frac{\nabla^{2} z_{i}}{z_{i}}\right) d t\right.}{z_{i}\left(1+G+d_{i} \frac{\nabla^{2} z_{i}}{z_{i}}\right) d t} \\
& =q_{i j}+\left(z_{i}\left(1+G+d_{i} \frac{\nabla^{2} z_{i}}{z_{i}}\right) d t\right)^{-1} q_{i j} \frac{\partial G}{\partial v} \delta u_{i j} d t \\
& +\left(z_{i}\left(1+G+d_{i} \frac{\nabla^{2} z_{i}}{z_{i}}\right) d t\right)^{-1} q_{i j}\left[d_{i}\left(\frac{\nabla^{2} z_{i j}}{z_{i j}}-\frac{\nabla^{2} z_{i}}{z_{i}}\right)\right] d t .
\end{aligned}
$$

Note that now we have expressed all quantities at time $t+d t$ in terms of quantitites at time $t$. The third term of Eq. (5.5) can be simplified further if we consider that:

$$
\begin{aligned}
& q_{i j}\left(\frac{\nabla^{2} z_{i j}}{z_{i j}}-\frac{\nabla^{2} z_{i}}{z_{i}}\right)=q_{i j}\left(\frac{\nabla^{2}\left(q_{i j} z_{i}\right)}{z_{i j}}-\frac{\nabla^{2} z_{i}}{z_{i}}\right) \\
& =q_{i j}\left[\frac{q_{i j} \nabla^{2} z_{i}}{z_{i j}}+\frac{2 \nabla q_{i j} \cdot \nabla z_{i}}{z_{i j}}+\frac{z_{i}}{z_{i j}} \nabla^{2} q_{i j}-\frac{\nabla^{2} z_{i}}{z_{i}}\right]==\frac{2 \nabla q_{i j} \cdot \nabla z_{i}}{z_{i}}+\nabla^{2} q_{i j},
\end{aligned}
$$


so that Eq. (5.5) becomes:

$q_{i j}+\left(z_{i}\left(1+G+d_{i} \frac{\nabla^{2} z_{i}}{z_{i}}\right) d t\right)^{-1} q_{i j} \frac{\partial G}{\partial v} \delta u_{i j} d t+\left(z_{i}\left(1+G+d_{i} \frac{\nabla^{2} z_{i}}{z_{i}}\right) d t\right)^{-1} d_{i}\left(\frac{2 \nabla q_{i j} \cdot \nabla z_{i}}{z_{i}}+\nabla^{2} q_{i j}\right) d t$

Inserting the first term of Eq. (5.7) in Eq. (5.2) clearly yields $s_{i}(\mathbf{x}, t)$. Inserting the second term of Eq. (5.7) in Eq. (5.2) yields a zero contribution, because, as in Chapter 3, $q_{i j}$ and $\left(\delta u_{i j}\right)^{2}$ are symmetrically distributed, but $\delta u_{i j}$ is anti-symmetrically distributed. Because $\left(\delta u_{i j}\right)^{2}$ are positionindependent, inserting the third term of Eq. (5.7) in Eq. (5.2) will yield a term proportional to:

$$
\sum_{j=1}^{N_{i}} \frac{2 \nabla\left(q_{i j}\left(\delta u_{i j}\right)^{2}\right) \cdot \nabla z_{i}}{z_{i}}+\nabla^{2}\left(q_{i j}\left(\delta u_{i j}\right)^{2}\right)=\frac{2 \nabla s_{i} \cdot \nabla z_{i}}{z_{i}}+\nabla^{2} s_{i}
$$

Therefore, if $s_{i}(\mathbf{x}, t)$ is position-indpendent at time $t$, then $\nabla s_{i}$ and $\nabla^{2} s_{i}$ are both zero, the third term in Eq. (5.7) will present a zero contribution to Eq. (5.2). We therefore find that $s_{i}(\mathbf{x}, t+d t)=$ $s_{i}(\mathbf{x}, t)=$ const., i.e., if initially position-independent, the phenotypic variance will constant in both space and time throughout, as in Eq. (3.24). 